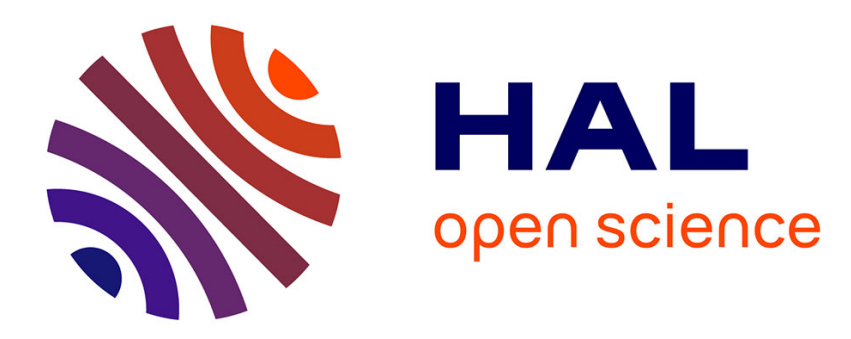

\title{
L'effet des arrêts maladie sur les trajectoires professionnelles
}

\author{
Thomas Barnay, Julie Favrot, Catherine Pollak
}

\section{To cite this version:}

Thomas Barnay, Julie Favrot, Catherine Pollak. L'effet des arrêts maladie sur les trajectoires professionnelles. Economie et Statistique / Economics and Statistics, 2015. hal-01297560

\section{HAL Id: hal-01297560 \\ https://hal.science/hal-01297560}

Submitted on 5 Apr 2016

HAL is a multi-disciplinary open access archive for the deposit and dissemination of scientific research documents, whether they are published or not. The documents may come from teaching and research institutions in France or abroad, or from public or private research centers.
L'archive ouverte pluridisciplinaire HAL, est destinée au dépôt et à la diffusion de documents scientifiques de niveau recherche, publiés ou non, émanant des établissements d'enseignement et de recherche français ou étrangers, des laboratoires publics ou privés. 


\title{
L'effet des arrêts maladie sur les trajectoires professionnelles
}

\author{
Thomas Barnay *, Julie Favrot **, Catherine Pollak ***
}

Rappel :

Les jugements et opinions exprimés par les auteurs

n'engagent qu'eux mêmes, et non les institutions auxquelles ils appartiennent, ni a fortiori l'Insee.
À la fin des années 2000, les taux d'emploi des personnes ayant des incapacités en France étaient de $46 \%$ contre $72 \%$ pour les personnes sans incapacités. Si l'on sait évaluer, pour l'Assurance maladie, le coût de l'indemnisation de l'incapacité temporaire de travail pour maladie (6,7 milliards d'euros soit 3,7\% des dépenses de santé en 2012), on ignore encore largement les effets de ces arrêts de travail sur les parcours professionnels.

Cette étude analyse l'effet des arrêts maladie - qui constituent une mesure de l'incapacité temporaire de travail - sur la dynamique des trajectoires professionnelles. Elle mobilise des données administratives de carrière et de dépenses de santé des salariés affiliés au Régime général (panel Hygie 2005-2008). Une typologie est mise en œuvre pour caractériser les transitions entre cinq états (emploi sans arrêt, emploi avec peu d'arrêts, emploi avec arrêts longs, chômage et inactivité). Le recours à un modèle multinomial dynamique à effets fixes permet d'estimer l'effet des épisodes d'arrêt maladie sur le chômage et l'inactivité d'une année à l'autre en distinguant la dépendance d'état de l'hétérogénéité inobservée.

Nous observons des trajectoires plus durablement dégradées (vers le chômage et l'inactivité notamment) pour les personnes ayant des arrêts de travail, en particulier lorsque ceux-ci sont dus à des longs épisodes de maladie. Cet effet est comparable pour les hommes et les femmes; le risque plus élevé d'inactivité après des arrêts longs chez les femmes s'expliquant principalement par l'hétérogénéité inobservée. Si les arrêts courts peuvent a priori s'interpréter comme un marqueur de sécurité de l'emploi, une fois l'hétérogénéité inobservée prise en compte, ils constituent également un facteur de risque pour les salariés dès lors que leur durée cumulée dépasse 30 jours. Les salariés semblent d'ailleurs intégrer ces risques de pénalités en limitant leur recours aux arrêts à la suite d'épisodes de chômage ou d'inactivité.

Codes JEL : J21, I14, C22.

Mots clés : arrêts maladie, trajectoires professionnelles, genre, modèle de transition.

\footnotetext{
* Créam, université de Rouen ; Erudite ; Tepp FR n`3435 - CNRS et Drees (thomas.barnay@univ-rouen.fr).

** BETA, université de Lorraine (julie.favrot@univ-lorraine.fr).

*** Drees (catherine.pollak@sante.gouv.fr).
}

Les auteurs tiennent à remercier Sylvie Blasco, Thibault Brodaty, Elise Coudin, Renaud Legal, et Julie Rochut pours leurs conseils et leur appui méthodologique, ainsi que Thierry Magnac pour leur avoir fourni ses programmes. Ils remercient également Pierre Blanchard, Pierre-Jean Messe, Jonathan Sicsic, Yann Videau, les participants aux Journées des économistes de la santé français, aux Journées de micro-économie appliquée, au séminaire du BETA, au séminaire Drees-Irdes sur les arrêts de travail, ainsi que deux rapporteurs anonymes pour leurs commentaires. Ils restent seuls responsables des limites ou inexactitudes de ce travail. Les points de vue exprimés dans cette étude doivent être considérés comme propres à leurs auteurs et ne sauraient en aucun cas engager la Drees. 
$\mathbf{E}^{n}$ n France, les dépenses d'indemnisation de l'incapacité (indemnités journalières pour maladie) représentaient 6,7 milliards d'euros en 2012, soit 3,7 \% des dépenses de santé (Le Garrec, Bouvet, 2013). La part de la richesse consacrée à l'indemnisation de l'invalidité et de l'incapacité (soit $0,7 \%$ du PIB pour chacune) y est inférieure à la moyenne des pays de l'OCDE. Pour autant, les personnes en incapacité ne sont pas davantage en emploi en France. Leur taux d'emploi se situe au niveau de la moyenne des pays de l'OCDE, et l'écart avec le taux d'emploi des personnes sans incapacités est de 25 points (OCDE, 2010, source $E U$-Silc 2007). Une partie des personnes en incapacité se trouve dans des situations de chômage ou d'inactivité. Des travaux récents ont montré qu'en France, les personnes ayant des maladies chroniques constituent un des groupes les plus défavorisés en termes de mobilité sur le marché du travail notamment du fait d'un risque élevé de transition de l'emploi vers le non emploi (Erhel, Guergoat-Larivière, 2013). Ces faits stylisés appellent à un renforcement des analyses des trajectoires professionnelles des personnes confrontées à des arrêts maladie.

Une littérature riche sur les liens entre état de santé et participation sur le marché du travail a mis en évidence le rôle de l'état de santé sur le passage à la retraite (Lumsdaine et Mitchell, 1999), sur les comportements d'offre de travail en général (Lindeboom, 2006) et de demande de travail (via par exemple des discriminations ressenties, $c f$. Bouvier et Jugnot, 2013). Des travaux français ont également relevé l'effet pénalisant de la mauvaise santé sur la participation au marché du travail (Tessier et Wolff, 2005) et la cessation anticipée d'activité (Barnay, 2005).

Cet article se situe dans la lignée de ces travaux en s'intéressant, pour la première fois en France, à l'effet des arrêts maladie sur les trajectoires professionnelles des salariés du secteur privé. Comparativement aux indicateurs de santé autodéclarée souvent mobilisés dans la littérature, les arrêts maladie présentent des propriétés intéressantes. Ils relèvent d'une expertise médicale et sont délivrés en cas d'incompatibilité entre l'état de santé de l'individu et l'exercice de son travail. Ils constituent ainsi une mesure pertinente de l'incapacité temporaire de travailler. Apparaissant comme un signal visible de l'état de santé supposé des salariés, ils sont susceptibles d'affecter directement les comportements de demande de travail (par des pénalités, discriminations, etc.).

L'article mobilise des données administratives (panel Hygie de 2005 à 2008) pour analyser les trajectoires professionnelles des salariés du privé âgés de 25 à 55 ans et confrontés à des arrêts maladie. Un modèle logistique multinomial est mis en œuvre afin de comparer les risques de trajectoires professionnelles descendantes selon que les périodes d'emploi précédentes aient été ou non caractérisées par des arrêts maladie. La modélisation dynamique à effets individuels fixes (Magnac, 2000) permet ensuite d'isoler l'effet des arrêts maladie de l'hétérogénéité inobservée constante dans le temps pouvant influencer à la fois la prise d'arrêts maladie et la situation sur le marché du travail. Les estimations renseignent sur l'impact des interruptions de travail liées à la santé sur l'intégration sur le marché du travail.

Dans cet article, nous dressons un état des lieux sur la littérature économique recouvrant le champ de l'effet de la santé, et plus spécifiquement des arrêts maladie, sur les trajectoires professionnelles, avant de présenter les données et la typologie des trajectoires professionnelles. Nous décrivons ensuite la stratégie économétrique et présentons les résultats de nos estimations. La dernière partie propose une discussion conclusive.

\section{Revue de littérature}

\section{Les effets négatifs de la santé sur les trajectoires professionnelles}

Cette étude traite des conséquences des absences dues à l'état de santé sur les trajectoires professionnelles. La littérature associée s'est formée autour du constat d'une forte corrélation entre état de santé et participation au marché travail. Une des hypothèses avancées stipule que les individus sont sélectionnés sur le marché du travail selon leur état de santé. Ce phénomène appelé healthy worker effect ou selection effect pourrait s'expliquer par des comportements d'offre de travail, dont les arbitrages sont affectés par une dégradation de l'état de santé, mais également par des comportements de demande de travail qui peuvent écarter les individus en mauvaise santé du fait de leur faible productivité réelle ou supposée et du coût associé à l'aménagement des postes.

Les travaux empiriques ont, dans un premier temps, beaucoup porté sur l'impact de la santé sur l'offre de travail au travers des comportements de départ à la retraite (Lindeboom, 2006). Bound et al. montrent, dès 1999, grâce à une 
spécification économétrique dynamique, l'impact d'une mauvaise santé sur un retrait précoce du marché du travail. Barnay (2005) dresse un constat similaire sur la population des seniors en France, faisant état d'un lien marqué entre dégradation de l'état de santé et cessation précoce d'activité, en particulier pour les ouvriers. Une mauvaise santé affecte également les trajectoires des travailleurs âgés de 30 à 54 ans qui ont un risque de chômage deux fois plus élevé que les personnes se déclarant en bonne santé (Jusot et al., 2008). Des analyses sur données de panel britanniques, contrôlant de l'hétérogénéité inobservée, concluent également à une relation positive entre l'état de santé général et mental et le salaire horaire chez les personnes en emploi (Contoyannis et Rice, 2001).

L'une des principales difficultés méthodologiques, interdisant souvent de conclure à un effet causal, réside dans la possibilité d'une causalité inverse du statut socioéconomique sur la santé. Plusieurs études ont ainsi mentionné l'effet néfaste de l'inactivité sur la santé perçue (Ross et Mirowsky, 1995) ou encore de celui du chômage sur le risque de mortalité (Mesrine, 2000). Pour autant, la simultanéité des effets causaux fait débat. Ainsi, Tessier et Wolff (2005) sont les premiers en France à recourir à un modèle à équations simultanées. Ils mettent en exergue uniquement l'impact négatif de l'état de santé dégradé sur l'offre de travail. En choisissant un choc exogène de perte d'emploi, Salm (2009) ne trouve aucun effet significatif sur l'état de santé perçu et sur les capacités à exercer des tâches de la vie quotidienne, dans les quatre années qui suivent la perte d'emploi. Haan et Myck (2009) ont recours à un modèle dynamique bivarié afin de saisir conjointement les corrélations entre mauvaise santé et non-emploi, à partir d'une population masculine âgée de 30 à 59 ans, et concluent quant à eux à un effet causal bidirectionnel.

\section{L'effet propre des arrêts maladie}

En étudiant l'impact des interruptions de travail liées à la santé sur les trajectoires professionnelles, cet article se situe dans la continuité de la littérature documentant l'effet de la santé sur le statut occupé par les individus sur le marché du travail.

Il s'en distingue toutefois par l'indicateur de santé utilisé. En effet, les précédentes études ont en commun de recourir à des indicateurs de santé perçue ou de santé objective. Les premiers souffrent de plusieurs biais notamment celui de justification dans la mesure où la déclaration d'un état de santé altéré peut être liée à une rationalisation du comportement de sortie du marché du travail (Bound, 1991). Par ailleurs, la comparabilité de ces indicateurs selon les catégories sociales, le genre et l'âge peut être compromise par l'existence de normes et d'attentes différentes face à la santé conduisant à des erreurs de mesure (Akashi-Ronquest et al., 2011 ; Etilé et Milcent, 2006; Shmueli, 2003). À l'opposé, le recours aux mesures objectives (décès, morbidité, maladies chroniques, etc.) n'est pas totalement satisfaisant. Bien qu'elles s'appuient sur le diagnostic médical, elles ont pour limite de ne donner qu'une information nécessairement incomplète de la santé. En outre, elles ne renseignent pas sur le degré d'adéquation entre état de santé et capacité de travail, ce qui peut se traduire par un biais de variable omise (Bound, 1991).

Pour ces raisons, les arrêts maladie peuvent être considérés comme un indicateur de santé objectivé. Ils présentent en effet l'avantage de fournir une mesure concrète et visible de l'incapacité temporaire de travail. Les jours d'absence représentent un coût direct pour l'employeur (baisse de productivité, éventuel recrutement pour remplacer le salarié, aménagement de poste, désorganisation de la production...). C'est pourquoi, même si le droit du travail protège les salariés contre les discriminations liées à l'état de santé (article L1132-1 du Code du Travail), il est possible que des arrêts de travail répétés ou longs finissent par leur porter préjudice (moindre promotion, non renouvellement de contrat, licenciement, etc. $)^{1}$.

Les travaux sur l'absentéisme considèrent d'ailleurs que les individus intègrent ces risques dans leurs choix de s'absenter. Les principaux modèles théoriques de l'absentéisme se placent dans un cadre où les absences procèdent d'un arbitrage entre travail et loisir (Allen, 1981). Dans ce cadre, l'utilité du loisir augmente a priori avec la maladie en raison d'un travail moins supportable ou de la baisse de productivité au travail ${ }^{2}$. Cependant, le coût d'opportunité de l'absence est d'autant plus élevé pour les salariés que

\footnotetext{
1. Le licenciement d'un salarié inapte est autorisé s'il est impossible de reclasser le salarié à l'issue d'un arrêt de travail, ou si en cas de maladie non professionnelle une absence prolongée ou des arrêt répétés perturbent le fonctionnement de l'entreprise et que l'employeur se trouve dans la nécessité de pourvoir à son remplacement définitif.

2. Cette hypothèse est valide lorsque l'effet de substitution domine l'effet de revenu. On peut aussi envisager qu'une dégradation de la santé s'accompagne d'une préférence accrue pour le travail du fait du coût des soins ou de l'assurance santé associée au travail.
} 
le risque de sanctions est important. Dans un contexte d'asymétrie d'information sur l'état de santé des salariés, le repérage des arrêts injustifiés représente toutefois un coût de contrôle important pour les employeurs. Ces derniers peuvent alors préférer au contrôle et à la sanction la fixation d'un salaire d'efficience afin d'empêcher les comportements d'abus (non shirking wage) (Barmby et al., 1994). Ces comportements d'absentéisme volontaire ${ }^{3}$ semblent cependant moins aisés dans le contexte français puisque les arrêts maladie doivent être justifiés auprès de l'employeur par une prescription médicale dès le premier jour. Néanmoins, la littérature empirique française a montré que le taux d'absences est effectivement plus faible parmi les salariés en contrats précaires (Inan, 2013 ; Chaupain-Guillot et al., 2007) et que les arrêts de courte durée se réduisent en période de chômage (Lê et Reynaud, 2007) tendant ainsi à confirmer un effet « disciplinant » du chômage.

Si la littérature sur les déterminants de l'absentéisme semble ainsi bien confirmer que les salariés intègrent le risque de pénalités dans leurs comportements de recours aux arrêts maladie, peu d'études (aucune à notre connaissance en France) se sont intéressées à l'effet réel des arrêts sur le risque de chômage ou d'éviction du marché du travail. Ce sont essentiellement les auteurs scandinaves qui ont étudié les conséquences des arrêts maladies sur la trajectoire professionnelle. Hansen (2000) montre un effet négatif de la prise d'arrêt maladie sur la trajectoire salariale en Suède mais seulement chez les femmes, chaque jour d'arrêt supplémentaire réduisant leur taux de salaire de $0,2 \%$. Une étude de Hesselius (2007) souligne que l'augmentation du nombre d'arrêts maladie et de leur fréquence accroît le risque de chômage. Plus récemment, Markussen (2012) établit qu'une augmentation du taux d'arrêts maladie se traduit par une baisse de la probabilité d'être en emploi deux ans plus tard. Pour les personnes restant en emploi, l'auteur observe également un impact négatif des arrêts maladie sur le salaire.

\section{Analyse descriptive des trajectoires}

\section{Données et échantillon}

L'analyse mobilise les quatre premières années du panel Hygie (2005 à 2008). Le système d'information sur les indemnités journalières
Hygie est issu d'un appariement de fichiers administratifs utilisés pour calculer le montant des indemnités journalières ainsi que le montant des pensions de retraite ${ }^{4}$. Il s'agit de la première base administrative française qui couple à la fois des informations sur les trajectoires professionnelles et les dépenses de santé des individus. Cette base contient l'ensemble des informations de carrière et de recours aux arrêts maladie indemnisés ${ }^{5}$ d'un échantillon d'environ 500000 salariés du privé (actifs ou retraités) âgés de 22 à 70 ans suivis sur plusieurs années ${ }^{6}$.

Pour les besoins de l'étude, l'analyse est restreinte aux personnes âgées de 25 à 55 ans. Il s'agit ainsi d'écarter des transitions atypiques liées aux difficultés d'insertion des jeunes et aux comportements spécifiques de retrait anticipé du marché de travail à l'approche de l'âge de la retraite (Barnay, Briard, 2009). Les personnes percevant des indemnités ou rentes pour accident du travail ou maladie professionnelle (ATMP) ont également été supprimées de l'échantillon car les déterminants de leurs trajectoires professionnelles sont susceptibles de différer sensiblement de celles des personnes ayant eu des arrêts maladie (notamment du fait des contraintes spécifiques des employeurs vis-à-vis des victimes d'ATMP). Le modèle économétrique mis en œuvre nécessite un panel cylindré, c'est pourquoi les individus, dont les données de carrière ne sont pas disponibles de 2005 à 2008, sont supprimés de l'échantillon. En outre, les salariés affiliés à un régime de sécurité sociale autre que le Régime général (ex : Régime social des indépendants, Mutualité sociale agricole) sont retirés de l'échantillon puisque l'information dont nous disposons sur leur carrière est incomplète. Au final, notre échantillon se compose de 247779 salariés du privé âgés de 25 à 55 ans en 2005 (pour le détail des filtres appliqués, voir annexe 1).

3. II a par exemple été démontré que les hommes suédois auraient tendance à recourir aux arrêts maladie pour suivre les compétitions sportives diffusées à la télévision (Skogman, Thoursie, 2004).

4. Ces données sont issues respectivement du Système national d'information inter-régimes de l'assurance maladie (SNIIR-AM) produit par la Caisse nationale de l'assurance maladie des travailleurs salariés (CNAMTS) et du Système national de gestion des carrières (SGNC) et du Système national statistiques prestataires (SNSP), gérés par la Caisse nationale d'assurance maladie (CNAV). La version utilisée dans l'article est celle des données actualisées au $1^{\text {er }}$ juillet 2014.

5. Les arrêts maladie d'une durée inférieure au délai de carence ( 3 jours dans le cas général) ne sont donc pas reportés dans la base Hygie.

6. II s'agit d'individus actifs ou retraités du régime général d'assurance vieillesse en 2005 et pour lesquels l'assurance maladie a enregistré au moins une prestation entre 2003 et 2005. 


\section{Une typologie de l'insertion professionnelle}

Les données de carrière distinguent les retraités des actifs mais ne contiennent pas de statut d'occupation précis. Afin de rendre compte du degré d'insertion professionnelle, nous construisons une typologie à partir des trimestres cotisés, des périodes assimilées (PA) au titre du chômage, de la maladie et de la maternité et des arrêts maladie.

L'enjeu de cette classification est la construction d'états distincts reflétant le statut individuel d'occupation afin d'obtenir un nombre de catégories nécessaire (pour ne pas regrouper des individus trop dissemblables du point de vue de leur position sur le marché du travail) et suffisant (pour disposer d'assez d'effectifs dans chacune d'entre elles). Cette typologie conduit à définir un statut d'occupation pour chaque année en cinq états (cf. encadré 1) :

État 1 : en emploi sans arrêt maladie ;

État 2 : en emploi avec des arrêts de courte durée; État 3 : en emploi avec des arrêts de longue durée (comprenant les congés maternité);

État 4 : chômage ou activité réduite ;

État 5 : en non emploi (chômage de longue durée et inactivité).

Encadré 1

\section{UNE TYPOLOGIE DES STATUTS SUR LE MARCHÉ DU TRAVAIL}

Le but de cette typologie est d'obtenir un classement des individus selon leur distance vis-à-vis du marché du travail. Les interruptions de travail sont intégrées dans les états afin de rendre compatible la typologie avec le recours à un modèle de transition.

Cette typologie est constituée de cinq états :

1. En emploi sans arrêt : individus qui ont cotisé quatre trimestres au titre du travail sans valider de périodes assimilées (PA) et qui n'ont pas connu d'épisode d'arrêts maladie. II s'agit de salariés principalement en emploi sur l'année ou qui ont travaillé avec un salaire suffisant pour valider quatre trimestres (soit au moins l'équivalent de 800 Smic horaire).

2. En emploi avec des arrêts de courte durée : individus qui ont cotisé quatre trimestres au titre du travail et qui ont eu moins de trois arrêts ou moins de 60 jours d'arrêts cumulés dans l'année. II s'agit de salariés principalement en emploi sur l'année (ou ayant travaillé avec un salaire suffisant pour valider quatre trimestres) qui ont eu des arrêts d'une durée inférieure au seuil permettant de valider une PA (le critère de validation d'une PA maladie est de 60 jours d'arrêts maladie indemnisés).

3. En emploi avec des arrêts de longue durée: individus qui ont cotisé au moins un trimestre au titre du travail et qui ont été en arrêt plus de 60 jours ou qui ont validé au moins une PA au titre de la maladie, d'un accident du travail ou d'une grossesse. Cette catégorie regroupe donc des personnes cumulant dans l'année des périodes de longue maladie et des périodes d'emploi. Notons toutefois que les trimestres sont comptabilisés chaque année mais ne sont pas ordonnés : il n'est donc pas possible de savoir si les périodes d'emploi sont antérieures ou consécutives aux périodes de maladie au sein d'une année.

De plus, il n'est pas possible d'identifier a priori les épisodes de congé maternité puisque les PA maladie, maternité et accident du travail sont confondues en une seule variable. Les individus ayant eu des indemnités journalières au titre d'un ATMP sont toutefois supprimés de l'échantillon. Un proxy est construit pour identifier les femmes ayant eu un congé maternité dans l'année. On considère que les femmes qui ont validé une période assimilée maladie/accident du travail/maternité alors qu'elles ne cumulent pas un nombre suffisant de jours d'arrêt maladie pour valider ce type de PA (critère 1 ) et qui ont entre 25 et 40 ans (critère 2) sont des femmes qui ont accouché dans l'année. En effet, la seule explication possible à cette validation de PA maladie/accident du travail/maternité est qu'il s'agit d'une PA maternité. La validation de PA maternité n'est pas soumise à un nombre de jours d'arrêt maladie, elle intervient automatiquement le trimestre où a lieu l'accouchement. Pour l'année 2005, on estime ainsi que $8,1 \%$ des femmes âgées de 25 à 40 ans étaient en congé maternité.

4. Au chômage ou en activité réduite : individus qui ont cotisé moins de quatre trimestres au régime général, sans que cette période ne soit complétée par une PA maladie. Cette catégorie intègre les chômeurs qui ont validé au moins une période assimilée au titre du chômage, et n'ont pas validé de PA maladie sur l'année. La validation d'une PA chômage nécessite d'avoir reçu une indemnisation au titre du chômage pendant 50 jours, même s'il est possible dans certains cas de valider une PA chômage pour des périodes de chômage non indemnisées. Sont également inclues les personnes qui ont moins de quatre trimestres cotisés sans toutefois avoir validé de PA chômage. En effet, certains chômeurs (au sens du BIT) peuvent ne pas avoir accumulé assez de droits pour avoir cette reconnaissance alors même qu'elles vivent une situation de chômage. Cet état comprend donc en plus des chômeurs indemnisés, les chômeurs non indemnisés et les individus partiellement inactifs.

5. En non emploi: individus qui n'ont pas du tout cotisé de trimestre au cours de l'année, c'est-à-dire qu'ils ont perçu moins de 200 fois le Smic horaire brut de l'année concernée. Cette catégorie regroupe donc les personnes qui n'ont pas travaillé de l'année et celles qui ont été présentes sur le marché du travail de manière marginale. Elle comprend donc les personnes inactives, celles qui ne peuvent pas travailler en raison de leur état de santé et les chômeurs de longue durée indemnisés ou non. 
Les états 2 et 3 intègrent explicitement les épisodes d'arrêt maladie selon leur intensité pour étudier l'impact graduel des interruptions de travail liées à la santé sur les transitions individuelles annuelles.

Afin de distinguer les deux états, un premier seuil de 60 jours d'arrêts est retenu; il constitue le seuil de validation d'une période assimilée au titre de la maladie. Il s'agit d'un seuil administratif au-delà duquel les arrêts maladie sont comptabilisés dans le calcul des droits à la retraite. Dans une seconde étape, la fixation du seuil fait l'objet de tests de sensibilité visant à identifier la durée la plus discriminante entre les états 2 et 3 dans les trajectoires professionnelles observées. Un seuil de 30 jours est alors arrêté.

Par ailleurs, les arrêts maladie couvrant deux années sont affectés à l'année caractérisée par la durée la plus longue et ce afin de ne pas confondre arrêts courts et longs ${ }^{7}$.

Enfin, sont réintégrées, au sein de l'état 3, les interruptions de travail de longue durée dues à des congés maternité afin de distinguer les effets des interruptions de travail de longue durée chez les femmes selon que celles-ci sont dues à la maternité ou à la maladie.

\section{Davantage d'arrêts longs chez les femmes en raison des congés maternité}

À l'aide de cette typologie, nous pouvons décrire les transitions individuelles observées entre 2005 et 2008 . Les états initiaux se distinguent substantiellement entre les hommes et les femmes, ces dernières étant plus nombreuses à être en marge du marché du travail (états 4 et 5) (cf. graphique I). Si les taux d'emploi sont plus élevés chez les hommes ${ }^{8}$, la proportion

7. Ces basculements à une autre année ne sont effectués que sur les parties d'arrêts d'une durée inférieure à 60 jours. Ceci permet de ne pas confondre les arrêts courts et les arrêts longs sans affecter la construction des autres états. Les arrêts longs sur deux années consécutives sont considérés comme une succession de deux états contenant des arrêts longs.

8. Les écarts de taux d'emploi entre hommes et femmes observés ici sont cohérents avec les données nationales en population générale. En 2005, d'après I'Insee, $58 \%$ des femmes et $69 \%$ des hommes de 15 à 64 ans étaient en emploi. Ces taux sont inférieurs de 10 points à ceux que nous obtenons à partir d'Hygie, ce qui peut s'expliquer par les taux d'emploi plus faibles des 15-25 ans et des plus de 55 ans exclus de notre échantillon.

\section{Graphique I}

Situation sur le marché du travail selon le genre en 2005

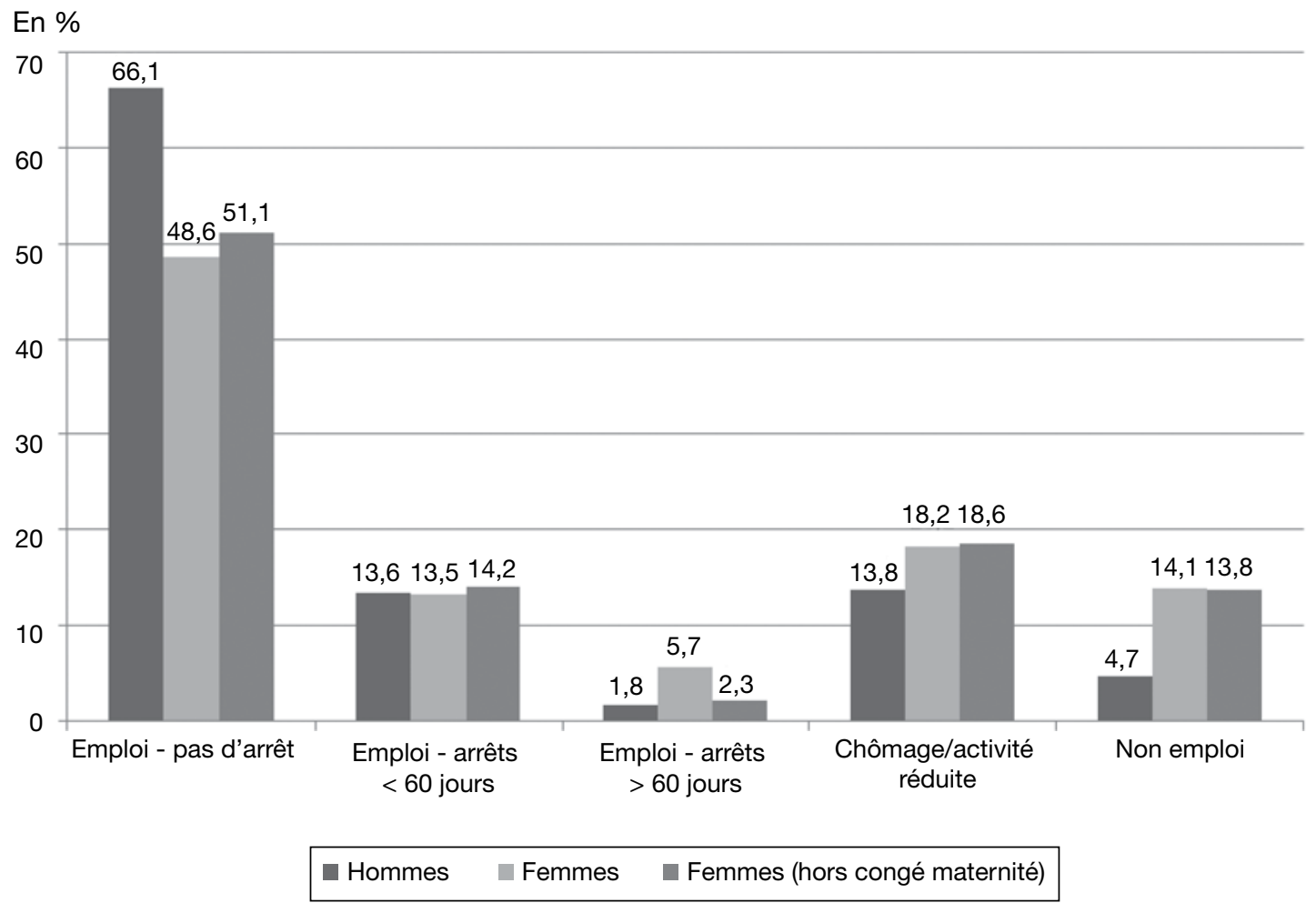

Lecture : 2,3 \% des femmes n'ayant pas eu de congé maternité étaient en emploi avec des arrêts supérieurs à 60 jours en 2005 Champ: hommes $(n=123$ 089) et femmes $(n=124$ 690) salariés du privé âgés de 25 à 55 ans (avec et sans congé maternité en 2005). Source: Hygie (2005-2008). 
d'hommes et de femmes en emploi et ayant recours dans l'année à des arrêts d'une durée cumulée inférieure à 60 jours est comparable (de l'ordre de $14 \%$ ). L'emploi associé à des arrêts longs concerne trois fois plus de femmes que d'hommes, mais cet écart s'explique par l'exclusion des individus en ATMP (davantage d'hommes que de femmes) et l'inclusion des femmes ayant eu un congé maternité : la part de femmes dans cet état est réduit de $5,7 \%$ à $2,3 \%$ lorsqu'on écarte les congés maternité.

\section{Des sorties de l'emploi plus fréquentes parmi les femmes ayant des arrêts maladie longs}

Une comparaison des probabilités brutes de transitions annuelles en fonction de la présence ou non d'arrêts maladie met en lumière des constats intéressants (tableaux 1 et 2 ). L'emploi avec de longs arrêts maladie (état 3 en $t$ - 1) semble a priori être le moins protecteur. $14,2 \%$ d'hommes et $18,9 \%$ de femmes ayant connu une longue période d'arrêt maladie en $t$ subissent une trajectoire dégradée menant au chômage ou à l'inactivité en $t+1$. Ces chiffres contrastent avec la proportion de trajectoires vers le chômage ou l'inactivité qui concerne $4,4 \%$ des hommes et $6,1 \%$ des femmes n'ayant pas eu d'arrêts. Depuis l'emploi avec des arrêts de courte durée, ces proportions passent à $5,9 \%$ et $7 \%$. Ainsi, les périodes d'arrêt courts semblent a priori nettement moins discriminantes que les interruptions longues pour maladie. De plus, l'examen des transitions à partir de trois premiers états met en évidence des différences systématiques entre les hommes et les femmes au détriment des trajectoires féminines.

Tableau 1

Transitions annuelles brutes entre états, population masculine

\begin{tabular}{|l|c|c|c|c|c|c|}
\cline { 2 - 7 } \multicolumn{1}{c|}{} & \multicolumn{7}{c|}{ En \% } \\
\hline Situation en $t-1$ & $\begin{array}{c}(1) \\
\text { Emploi } \\
\text { sans arrêts }\end{array}$ & $\begin{array}{c}(2) \\
\text { Emploi } \\
\text { arrêts }<60 j\end{array}$ & $\begin{array}{c}(3) \\
\text { Emploi } \\
\text { arrêts }>60 j\end{array}$ & $\begin{array}{c}\text { (4) } \\
\text { Chômage, } \\
\text { activité réduite }\end{array}$ & $\begin{array}{c}\text { (5) } \\
\text { Non emploi }\end{array}$ & \multicolumn{1}{c|}{ Total } \\
\hline (1) Emploi sans arrêts & 82,6 & 11,7 & 1,4 & $3,7^{*}$ & 0,7 & 100 \\
(2) Emploi arrêts $<60 \mathrm{j}$ & 59,1 & 30,2 & 5,0 & 4,8 & 1,1 & 100 \\
(3) Emploi arrêts $>60 j$ & 37,9 & 23,8 & 24,0 & 8,5 & 5,7 & 100 \\
(4) Chômage, activité réduite & 29,4 & 4,7 & 0,8 & 52,1 & 12,9 & 100 \\
(5) Non emploi & 11,0 & 1,3 & 0,7 & 34,1 & 53,0 & 100 \\
\hline Total & 68,7 & 13,0 & 2,2 & 11,4 & 4,8 & 100 \\
\hline
\end{tabular}

Lecture : parmi les hommes en emploi sans arrêt l'année en $\mathrm{t}-1$ (état 1), 3,7 \% transitent vers le chômage ou l'activité réduite en $\mathrm{t}$ (état 4). Champ : salariés du privé âgés de 25 à 55 ans $(n=124$ 199).

Source: Hygie (2005-2008).

Tableau 2

Transitions annuelles brutes entre états, population féminine (hors congés maternité)

En \%

\begin{tabular}{|c|c|c|c|c|c|c|}
\hline \multirow[b]{2}{*}{ Situation en $t-1$} & \multicolumn{6}{|c|}{ Situation en $t$} \\
\hline & $\begin{array}{c}\text { (1) } \\
\text { Emploi } \\
\text { sans arrêts }\end{array}$ & $\begin{array}{c}(2) \\
\text { Emploi } \\
\text { arrêts < 60j }\end{array}$ & $\begin{array}{c}(3) \\
\text { Emploi } \\
\text { arrêts > 60j }\end{array}$ & $\begin{array}{c}\text { (4) } \\
\text { Chômage, } \\
\text { activité réduite }\end{array}$ & $\begin{array}{c}(5) \\
\text { Non emploi }\end{array}$ & Total \\
\hline (1) Emploi sans arrêts & 77,5 & 14,4 & 2,0 & $5,2^{*}$ & 0,9 & 100 \\
\hline (2) Emploi arrêts < 60j & 53,4 & 33,5 & 6,1 & 5,7 & 1,3 & 100 \\
\hline (3) Emploi arrêts > 60j & 32,9 & 23,1 & 25,1 & 10,0 & 8,9 & 100 \\
\hline (4) Chômage, activité réduite & 28,3 & 5,8 & 1,2 & 52,0 & 12,6 & 100 \\
\hline (5) Non emploi & 7,7 & 1,3 & 0,4 & 23,4 & 67,2 & 100 \\
\hline Total & 55,7 & 14,0 & 2,7 & 15,8 & 11,8 & 100 \\
\hline
\end{tabular}

Lecture : parmi les femmes en emploi sans arrêt l'année en $\mathrm{t}-1$ (état 1), 5,2 \% transitent vers le chômage ou d'activité réduite en $\mathrm{t}$ (état 4). Champ : salariées du privé âgées de 25 à 55 ans n'ayant pas eu de congé maternité entre 2005 et $2008(n=107985)$.

Source : Hygie (2005-2008). 


\section{Davantage de retours à l'emploi sans arrêt suite à des congés maternité}

Les transitions professionnelles des femmes caractérisées par des arrêts longs l'année précédente sont particulièrement sensibles à la nature de l'arrêt (maladie ou maternité) (cf. graphique II). Lorsque l'arrêt long est lié à la maladie, un tiers des femmes se retrouvent un an après en emploi sans arrêt. Cette proportion atteint $54 \%$ si les femmes ont été arrêtées plus de 60 jours en raison d'une maternité.

Ces résultats bruts ne permettent cependant pas de conclure à un effet des arrêts maladie sur les trajectoires ultérieures, puisqu'ils ne tiennent pas compte des différences de structure des populations situées dans les différents états initiaux (âge, état de santé, secteurs d'activité, etc.) ni de l'hétérogénéité inobservée (stabilité, attachement au travail, motivation, compétences, etc.) susceptibles d'expliquer largement les changements de situation sur le marché du travail. Pour identifier finement ces paramètres, des modèles de transition dynamique sont mis en œuvre afin de distinguer l'hétérogénéité individuelle (observée et non observée) et la dépendance d'état, c'est-à-dire l'effet propre du passage par un état en $t-1$ sur la situation en $t$.

\section{Méthodologie économétrique}

Cet article met en ouvre un modèle dynamique de transition à partir de données administratives en panel pour retracer l'effet des épisodes d'arrêts maladie sur les trajectoires professionnelles des salariés. Les modèles de transition présentent l'intérêt de capter l'effet du passage par un état sur une multiplicité de transitions possibles sur le marché du travail en distinguant la dépendance d'état de l'hétérogénéité observée et inobservée. L'évaluation de l'impact du passage dans un état une année sur les états futurs suppose d'intégrer dans la variable explicative une valeur retardée de la variable dépendante, ou en d'autres termes d'évaluer la dépendance d'état. Heckman (1981) souligne les difficultés liées à l'observation de la dépendance d'état. L'hypothèse, selon laquelle les états passés constituent des prédicteurs des états futurs, renvoie à deux effets. Naturellement, les événements rencontrés durant le parcours des individus affectent la probabilité de faire face à d'autres évènements (vraie dépendance d'état). Cependant, les transitions empiriques observées peuvent aussi être liées à des caractéristiques individuelles non observables (fausse dépendance d'état).

\section{Graphique II}

Transitions des femmes consécutives à un arrêt long (congés maternité vs maladie)

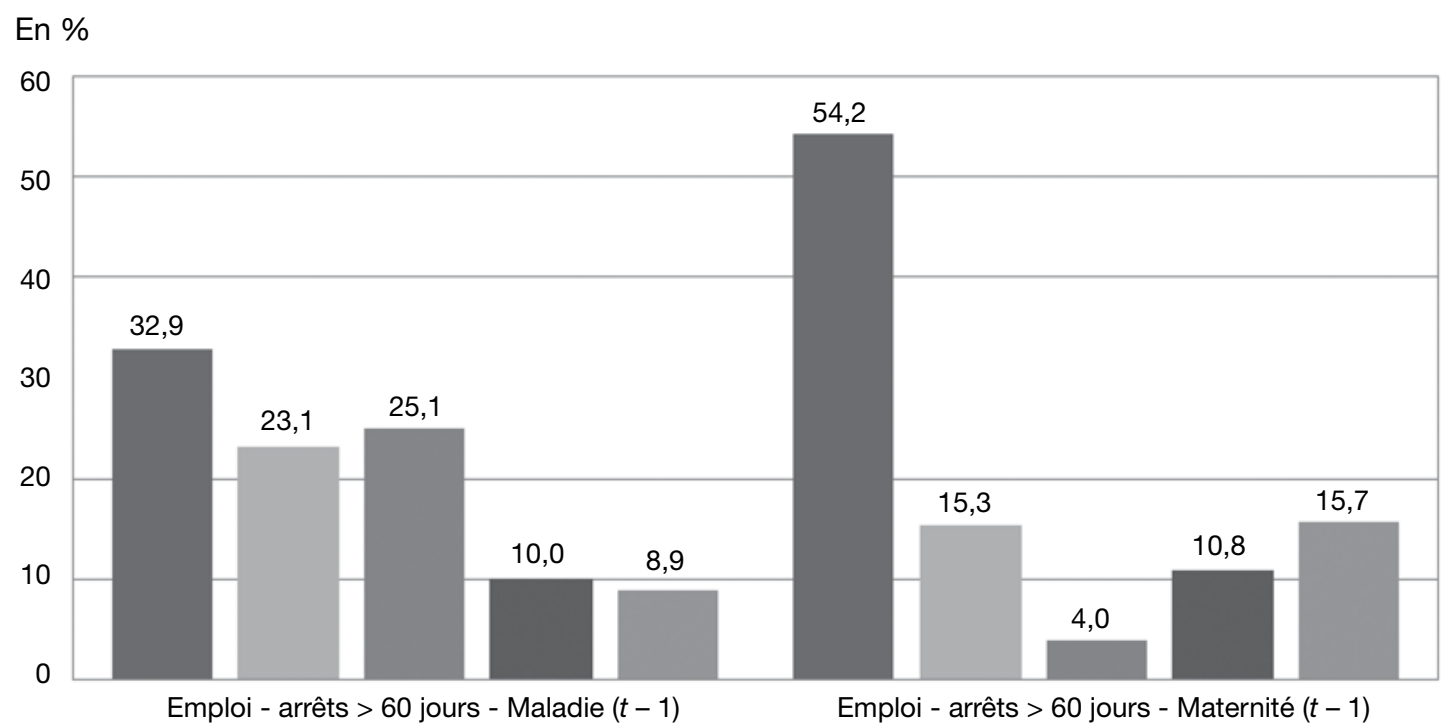

Emploi - pas d'arrêt (t) $\square$ Emploi - arrêts $<60$ jours (t) $\square$ Emploi - arrêts $>60$ jours (t) $\square$ Chômage/activité réduite (t) $\square$ Non emploi (t)

Lecture : parmi les femmes en emploi ayant eu un arrêt long (> à 60 jours) en t - 1 en raison d'un congé maternité, 54,2 \% ont transité vers l'emploi sans arrêt en $\mathrm{t}$.

Champ : femmes salariées du privé âgées de 25 à 55 ans, hors ATMP.

Source : Hygie 2005-2008. 
Il convient par conséquent de démêler la dépendance d'état de l'hétérogénéité inobservée.

En économie du travail, les travaux de Magnac (2000) constituent une référence dans la mise en œuvre de modèles multinomiaux dynamiques. Afin d'évaluer l'impact des programmes de formation destinés aux jeunes actifs, il a recours à plusieurs modèles dynamiques dans lesquels sont étudiées les transitions entre six états sur le marché du travail. Pour s'affranchir du biais soulevé par Heckman (1981), Magnac inclut des effets individuels fixes dans la modélisation d'un modèle multinomial dynamique. La même année, Honoré et Kyriazidou proposent une alternative à ce modèle, avec une spécification économétrique permettant d'ajouter des variables explicatives qui varient dans le temps alors que le modèle de Magnac n'autorise qu'une seule variable explicative, à savoir la valeur retardée de la variable dépendante. Cette stratégie d'estimation s'avère notamment utile pour prendre en compte l'effet des variations conjoncturelles sur les transitions des contrats temporaires vers l'emploi stable (Givord et Wilner, 2009). Le modèle de Magnac est repris ensuite par Beck et Kamionka (2012) pour évaluer la mobilité des salariés entre catégories socioprofessionnelles, statuts et secteurs d'activité. D'autres études recourent à des effets aléatoires, à l'instar d'Havet (2006) dans une analyse de l'insertion professionnelle des jeunes tout en traitant le problème des conditions initiales en utilisant la méthode proposée par Wooldridge (2005). Enfin, Blasco et Givord (2010) proposent d'intégrer l'effet de la durée du passage dans un état en mettant en œuvre un modèle « multi-états multi-épisodes ».

Cet article privilégie le modèle de Magnac en estimant un modèle dynamique de transition à partir de données administratives en panel (base Hygie 2005-2008) pour retracer les trajectoires professionnelles des salariés cotisants au régime général âgés de 25 à 55 ans. Le modèle de Magnac permet d'isoler la dépendance d'état (l'effet propre des arrêts maladie) des facteurs d'hétérogénéité observés et inobservés fixes dans le temps pouvant affecter à la fois l'état de santé, le recours aux arrêts maladie, et la situation sur le marché du travail (telles que la catégorie socioprofessionnelle, la stabilité de l'emploi, les préférences des individus au regard de l'arbitrage travail/loisir ou encore l'aversion au risque) sur les trajectoires. Sa stratégie d'estimation par maximum de vraisemblance conditionnelle (dépendant d'une statistique suffisante et non des effets fixes individuels) permet de résoudre le problème des conditions initiales soulevé par Heckman (1981) et garantit la robustesse de l'estimation à toute spécification de l'hétérogénéité inobservée (Brodaty, 2008). C'est pour cette raison qu'il est préféré à un modèle à effets aléatoires tel que proposé par Havet (2006). Cependant, en raison d'un problème de paramètres incidents, le modèle de Magnac contraint à la sélection d'une population qui a changé de statut entre 2006 et 2007 (population que nous qualifions de «movers ») (cf. encadré 2).

Ce modèle permet également d'obtenir des paramètres consistants avec un nombre de périodes réduit (les quatre années du panel sont suffisantes) sans intégrer de covariables dans l'estimation. Même s'il existe des modèles permettant d'ajouter des facteurs explicatifs variables dans le temps (Honoré, Kyriazidou, 2000), il semble peu probable que leur non prise en compte constitue une source de biais dans cette étude. Les variations conjoncturelles, par exemple, peuvent affecter à la fois les arrêts maladie et le degré d'insertion professionnelle mais pas dans le même sens. Une hausse du chômage est de nature à accroître le risque de trajectoires professionnelles dégradées mais réduira, dans le même temps, le recours aux arrêts maladie courts (Lê et Raynaud, 2007). Ne pas en tenir compte ne peut donc que conduire à sous-estimer l'ampleur des liens causaux mis en évidence. Au niveau des facteurs individuels, les principaux déterminants conjoints des arrêts maladie et du statut sur le marché du travail sont a priori fixes dans le temps.

De la même façon, ce modèle à effets fixes ne tient pas compte des variations de l'état de santé susceptibles d'influer sur la trajectoire professionnelle et indépendantes des arrêts maladie. Il n'établit donc pas d'estimation de l'impact causal des arrêts maladie à variation de santé équivalente. En effet, l'objectif de cette étude n'est pas d'apprécier l'effet des arrêts maladie sur les trajectoires selon que ceux-ci soient plus ou moins justifiés par l'état de santé. En revanche, le modèle permet d'estimer le risque chômage ou d'inactivité pour des personnes ayant connu des interruptions de travail pour incapacité à caractéristiques fixes dans le temps équivalentes. Une transition vers des arrêts de travail plus longs peut par conséquent simplement relever d'une dégradation de leur état de santé (et non de l'impact causal d'arrêts courts sur des arrêts longs). En outre, une transition vers le chômage ou l'inactivité pourra s'être accompagnée d'une dégradation de l'état de santé. Enfin, même si la composante dynamique inobservée de l'état de 
santé n'est pas considérée dans ce modèle, on peut raisonnablement faire l'hypothèse qu'un état de santé susceptible d'affecter le statut d'emploi se traduira par des arrêts maladie.

Afin d'analyser les trajectoires entre les cinq situations sur le marché du travail définies par la typologie, nous mettons en œuvre, dans un premier temps, un modèle logit multinomial reposant sur un simple contrôle de l'hétérogénéité observée et des conditions initiales (approximées à travers l'âge d'entrée sur le marché du travail) (encadré 2). Ces dernières peuvent être déterminantes dans la suite de la trajectoire professionnelle et leur effet ne doit pas être confondu avec celui de l'événement « interruption de travail liée à la santé » que nous souhaitons isoler'. Dans un second temps, nous mobilisons le modèle logit multinomial dynamique à effets fixes de Magnac (encadré 3). La comparaison des deux modèles permet d'estimer dans quelle mesure la prise en compte de l'hétérogénéité inobservée atténue la mesure du risque associé à ces trajectoires.

\section{Résultats}

I (contrôlant de l'hétérogénéité observée) sont comparés à ceux du modèle multinomial à effets fixes de Magnac (tenant compte de l'hétérogénéité inobservée) en stratifiant selon le genre. Précisons cependant que le modèle à effets fixes sélectionne une population particulière (les personnes qui changent de statut entre 2006 et 2007 qualifiées de " movers », cf. annexe 2$)^{10}$. Dans un premier temps, on examine les transitions professionnelles des hommes (tableau 3) et des femmes hors congés maternité (tableau 4). Afin de faciliter la lecture et

9. Pour illustrer ce propos, imaginons que les personnes peu qualifiées aient à la fois un risque plus élevé de chômage et de mauvaise santé que les personnes qualifiées. Si nous observons un risque plus élevé de chômage en $\mathrm{t}$ chez les personnes ayant eu des arrêts maladie en $\mathrm{t}-1$, il peut être lié aux conditions initiales (les mêmes individus ont dès le départ des risques de chômage et de maladie plus élevés) plutôt qu'à des épisodes antérieurs de maladie (fausse dépendance d'état).

10. La restriction aux "movers" a pour effet mécanique de réduire l'ampleur des coefficients associés aux transitions estimées. En effet, ils ont pour spécificité d'être moins nombreux à avoir des transitions diagonales et plus nombreux à avoir des changements d'état (cf. annexe 2). Leurs probabilités de transitions sont donc estimées par rapport à une référence qui est plus élevée par construction (le retour à l'état 1 pour les personnes provenant d'autres états - première colonne, et le départ vers d'autres états pour les personnes provenant de l'état 1 - première ligne). La comparaison des deux modèles tient donc à la fois à cette différence de champ et à la prise en compte de l'hétérogénéité inobservée. En revanche, la restriction aux " movers " affecte uniformément les différents sous-groupes. La modification des écarts de coefficients entre les groupes lorsque l'on passe au modèle à effets fixes tient donc bien à la prise en compte de l'hétérogénéité inobservée qui distingue les hommes, les femmes sans congé maternité, et les femmes ayant eu des congés maternités.

Encadré 2

\section{MODÈLE LOGIT MULTINOMIAL DYNAMIQUE}

Le premier modèle prend la forme d'un logit multinomial non ordonné sur données empilées (pooled model). Pour identifier ce type de modèle, il est nécessaire de définir un état de référence (généralement le plus répandu), ici l'état « en emploi sans arrêt » (état 1). Pour cet état de référence $j=1$, on pose $\beta_{1}=0$. Les paramètres estimés $\beta_{j}$ s'interprètent alors en référence à l'état 1 .

Le modèle s'écrit de la manière suivante :

$y_{i j}^{\star}=\sum_{k=0}^{j-1} \delta_{k j} 1\left(y_{i, t-1}=k\right)+\beta_{j} X_{i}+\varepsilon_{i j}$

où :

- $y_{i j}^{*}$ est une variable latente qui décrit la propension d'un individu $i$ à occuper l'état $j$;

- $\delta_{k j}$ exprime l'impact du passage dans un état $k$ en $t-1$ sur la probabilité d'être dans l'état $j$ en $t$, cette variable est un paramètre de dépendance d'état qui suit un processus de Markov d'ordre 1;
- $\varepsilon_{i j}$ représente le terme d'erreur du modèle qui suit une loi de Gompertz.

Plusieurs variables de contrôle, présentes dans le vecteur $X_{i}$, permettent de dissocier la dépendance d'état de l'hétérogénéité observée. Pour ce faire, nous incluons un contrôle de l'hétérogénéité temporelle (effet de période ou conjoncturel) par le biais d'une indicatrice de la date à laquelle l'individu a effectué sa transition. Nous approchons les conditions initiales par l'âge d'entrée sur le marché du travail, en faisant I'hypothèse que le moment d'entrée sur le marché du travail reflète largement les différences initiales entre individus (préférences, motivations, niveau de diplôme, etc.). Enfin, dans cette première étape, nous captons l'hétérogénéité individuelle à travers plusieurs variables démographiques (âge), d'état de santé (consommation de soins en $t-1$ en quintiles, reconnaissance administrative d'une affection de longue durée «ALD » en $t-1$ ), et de conditions de travail (secteur d'activité en $t-1$ ). 
l'interprétation des résultats, les coefficients issus des estimations sont présentés sous forme d'odds ratios. Un odds ratio significativement supérieur à 1 (respectivement inférieur à 1) signifie que le passage par l'état $k$ en $t$-1, plutôt que par l'emploi sans arrêt, augmente (respectivement réduit) les chances d'arriver à l'état $k$ ' en $t$ plutôt que de retourner à l'emploi sans arrêt. Dans un second temps, on compare les trajectoires des femmes hors congés maternité à celles de l'ensemble des femmes (annexe 3A). L'inclusion des congés maternité pour ces dernières augmente le nombre de femmes en arrêt long (état 3).

\section{L'effet pénalisant des arrêts maladie sur les sorties de l'emploi}

Les résultats des modèles multinomiaux corroborent l'effet pénalisant des arrêts maladie observé à partir des transitions brutes (tableaux 3 et 4). Ils font cependant apparaître un risque de trajectoires dégradées y compris

Encadré 3

\section{MODĖLE LOGIT MULTINOMIAL À EFFETS FIXES}

Le modèle logit multinomial dynamique à effets fixes de Magnac (2000) contrôle de l'hétérogénéité inobservée par des effets fixes, mettant ainsi pleinement à profit la dimension longitudinale des données.

Avec ce modèle à effets fixes, l'expression de la probabilité pour un individu $j$ de se retrouver dans l'état $i$ devient (Givord et Wilner, 2009) :

$P\left(y_{i, t}=j \mid y_{i, t-1}=k ; \alpha ; \delta\right)=\frac{e^{\delta_{k j}+\alpha_{i j}}}{1+\sum_{l \neq 0} e^{\delta_{k l}+\alpha_{i j l}}}$

On remarque alors que la probabilité individuelle ainsi exprimée dépend d'un terme de dépendance d'état commun à tous les individus mais aussi d'un terme d'hétérogénéité individuelle.

Dans ce modèle, on aura alors une variable latente de la forme suivante :

$y_{i j t}^{*}=\sum_{k=0}^{j-1} \delta_{k j} 1\left(y_{i, t-1}=k\right)+\varepsilon_{i j t}$

Cette variable latente décrit la propension d'un individu $i$ à occuper un état $j$ à la période $t$. Le terme d'erreur se décompose en deux parties :

$\varepsilon_{i j t}=\alpha_{i j}+u_{i j t}$

où $\alpha_{i j}$ représente la propension d'un individu $i$ à occuper un état $j$ contrôlant ainsi de l'hétérogénéité individuelle et $u_{i j t}$ un terme d'erreur suivant une loi des valeurs extrêmes (loi de Gompertz).

Les paramètres à estimer sont les $\delta_{k j}$ qui représentent la propension d'un individu à aller vers l'état $j$ sachant qu'il était dans l'état $k$ précédemment. Ces paramètres de dépendance d'état suivent un processus markovien d'ordre 1.
La présence d'effets fixes pose un problème de paramètres incidents (incidental parameter problem) qui rend l'estimateur non convergent lorsqu'on utilise la méthode du maximum de vraisemblance. Pour surmonter ce problème, Magnac (ibid) propose une statistique suffisante afin de ne plus faire dépendre l'estimation des effets fixes $\alpha_{i j}$. Cette statistique suffisante fait intervenir l'état initial $y_{1}$, de l'état final $y_{T}$ et de la somme des états intermédiaires $n_{i k}=\sum 1\left(y_{i t}=k\right)$.

En raison de l'adoption de cette statistique suffisante, seuls les individus ayant connu une transition entre $t=2$ et $T-1$ contribuent à la vraisemblance, l'estimation se fait donc sur les movers. Dans le cas présent, seules les personnes qui ont changé d'état entre 2006 et 2007 participent à la vraisemblance.

Les coefficients ainsi estimés s'interprètent comme des rapports de probabilité :

$\delta_{k j}-\delta_{0 j}=\log \frac{\left(\frac{P\left(y_{i t}=j \mid y_{i, t-1}=k ; \alpha\right)}{P\left(y_{i t}=0 \mid y_{i, t-1}=k ; \alpha\right)}\right)}{\left(\frac{P\left(y_{i t}=j \mid y_{i, t-1}=0 ; \alpha\right)}{P\left(y_{i t}=0 \mid y_{i, t-1}=0 ; \alpha\right)}\right)}$

Les paramètres $\delta_{k j}$ ne sont pas identifiables pour tous les états, c'est pourquoi on a un état de référence $k=0$ pour lequel tous les paramètres relatifs à cet état seront nuls. On aura également $\alpha_{i 0}=0$. Ici l'état de référence est l'état 1 , comme dans le premier modèle. Ainsi, si le paramètre de dépendance d'état est positif $\left(\delta_{k j}>0\right)$ et significatif, l'interprétation du paramètre sera la suivante : "Le fait d'avoir été dans l'état $k$ plutôt que dans l'état "en emploi sans arrêt " (état 1) en $t-1$ augmente les chances d'être en $j$ plutôt que dans l'état « en emploi sans arrêt » (état 1).

Comme, il s'agit d'un modèle semi-paramétrique, il n'est pas nécessaire d'émettre des hypothèses sur la loi des effets individuels, ce qui nous affranchit des hypothèses contraignantes des modèles à effets aléatoires. 
pour les personnes ayant eu des arrêts maladie d'une durée cumulée inférieure à 60 jours. Dès lors que l'activité professionnelle est marquée par des arrêts maladie (états 2 et 3 ), comparativement aux personnes qui n'ont pas eu d'arrêt, on observe un accroissement significatif du risque de transition descendante plutôt qu'un retour à l'emploi ininterrompu (état 1).

L'introduction d'effets fixes ne remet pas en question l'effet pénalisant des arrêts maladie, et cela quelles que soient leur intensité et la significativité globale des transitions. Les résultats confirment que les arrêts longs pénalisent plus fortement les trajectoires professionnelles de court terme que les arrêts courts. Si l'on s'en tient à l'examen exclusif des modèles à effets fixes, le risque de transition vers le non emploi (plutôt que vers le retour à l'emploi sans arrêt) suite à des arrêts longs est multiplié par plus de 8 comparé au risque de non emploi des individus n'ayant pas eu d'arrêts maladie. Ce type d'arrêts accroît cependant également le risque de transition vers le chômage; associé à des odds ratios de 3,4 chez les hommes et de 3,5 chez les femmes.

De façon générale, les arrêts maladie de courte durée constituent plus souvent un état transitoire que les arrêts de longue durée, tout particulièrement lorsqu'est prise en compte l'hétérogénéité inobservée. Même si l'effet des arrêts courts est globalement moins pénalisant que celui des arrêts longs, il contribue néanmoins à accroître le risque de chômage et d'inactivité. L'effet est néanmoins de faible ampleur: les hommes avec des arrêts maladie courts l'année précédente ont un risque de 1,2 d'être au chômage et 1,3 d'être en non emploi l'année suivante plutôt qu'en emploi sans arrêt. Pour les femmes (hors congés maternité), ces risques sont de 1,2 pour les deux transitions.

\section{Tableau 3}

Modèles de transition, population masculine (seuil à 60 jours)

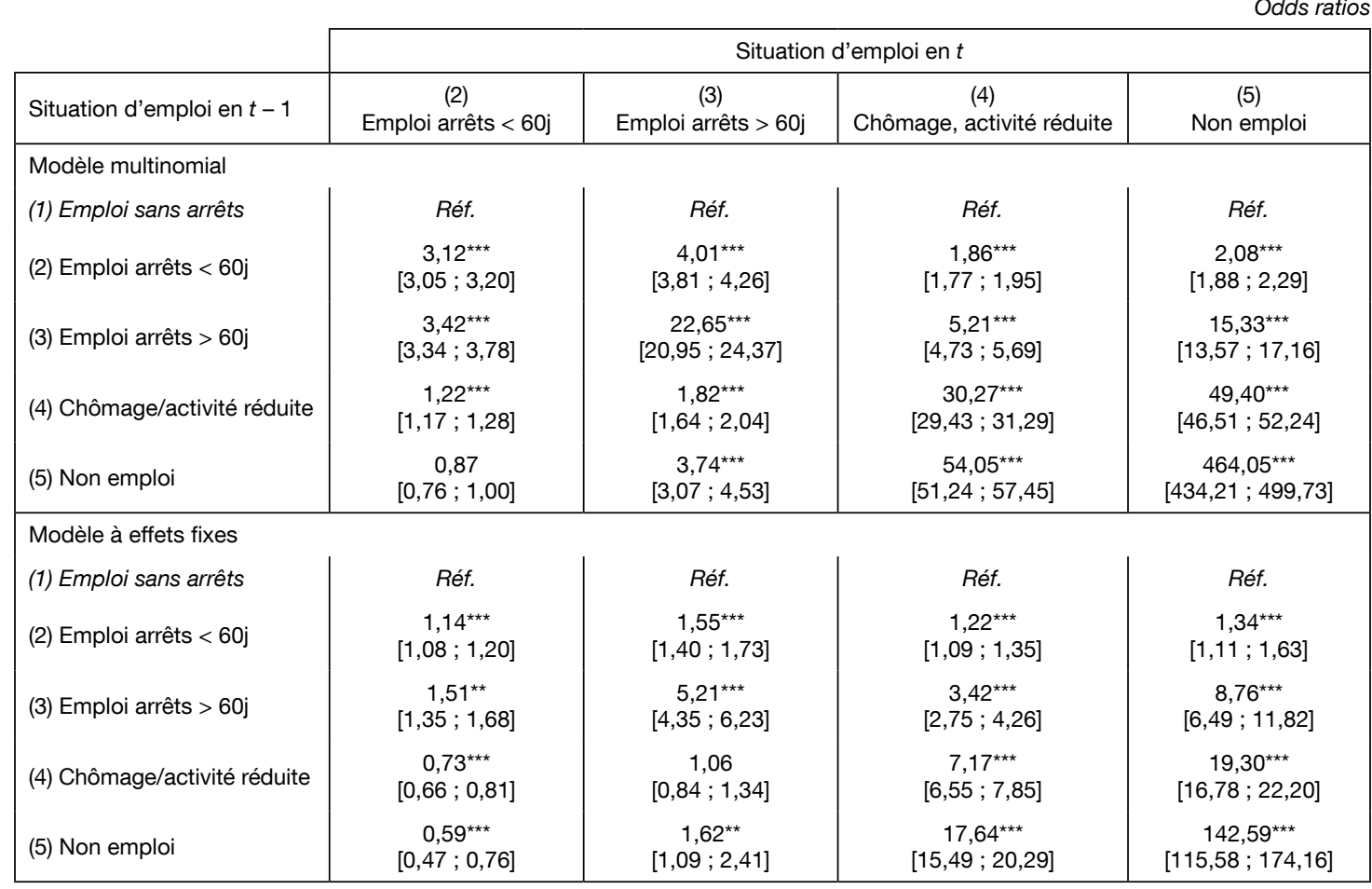

Note : afin de faciliter la lecture et l'interprétation des résultats, les chiffres des tableaux 3 et 4 sont présentés sous forme d'odds ratios. Les odds ratios donnent une approximation du risque relatif de transition de l'état $k$ en $t-1$ (plutôt que l'état en "Emploi sans arrêt») vers l'état $j$ en $t$ en référence à l'état en «Emploi sans arrêt ». Si l'odds ratio est significativement supérieur (ou inférieur) à 1 alors l'impact est positif (ou négatif), toutes choses égales par ailleurs.

Lecture : dans le modèle à effets fixes, un homme ayant connu peu d'arrêts maladie, par rapport à un homme en emploi sans arrêts, a 1,34 fois plus de chances d'être en non emploi l'année suivante plutôt qu'en emploi sans arrêts au seuil de 0,1\%. Trois seuils de significativité sont calculés : ${ }^{*}<0,05 ;{ }^{* *}<0,01 ;{ }^{* *}<0,001$. Les odds ratios estimés à partir du modèle multinomial sont contrôlés de l'hétérogénéité observée (ALD en $\mathrm{t}-1$, dépense totale de soins en $\mathrm{t}-1$, âge en 2005 , âge d'entrée sur le marché du travail, bénéficiaire CMU-C en $\mathrm{t}-1$, secteurs d'activité en $\mathrm{t}-1$ et indicatrice temporelle de transition).

Champ : modèle multinomial : hommes âgés de 25 à 55 ans ( $n=369$ 967, données empilées) ; modèle à effets fixes : hommes âgés de 25 à 55 ans « movers » entre 2006 et $2007(n=37567)$.

Source : Hygie (2005-2008). 
Des arrêts maladie de courte durée semblent par ailleurs prédisposer les individus à en avoir davantage; le risque de transition vers l'emploi associé à des arrêts longs (état 3) apparaît plus élevé que le retour à l'emploi sans arrêt comparé aux individus n'ayant eu aucun arrêt (1,6 pour les hommes et les femmes).

La mesure de l'intensité des arrêts maladie est conditionnée au choix du seuil permettant de distinguer les états 2 et 3 de la typologie adoptée. Les résultats des estimations précédentes montrent en effet que même les arrêts de moins de 60 jours accroissent significativement le risque de chômage et d'inactivité chez les hommes et les femmes. Afin de tester la sensibilité de ces résultats au seuil retenu, plusieurs seuils sont testés (de 50, 40 et 30 jours). Les résultats changent significativement à partir d'un seuil de 30 jours (annexe 3B et 3C). Ils mettent en évidence que les arrêts d'une durée cumulée inférieure à 30 jours n'ont pas d'effet sur le risque d'inactivité. Ces arrêts relativement courts ont néanmoins un effet positif et significatif sur le risque de chômage, même si l'effet est de faible ampleur (proche de 1). Les arrêts d'une durée supérieure à un mois accroissent quant à eux non seulement le risque de chômage mais aussi le risque d'inactivité.

\section{L'hétérogénéité inobservée fixe dans le temps explique les différences entre les hommes et les femmes}

Deux phénomènes différencient hommes et femmes dans le modèle multinomial. Les arrêts longs apparaissent plus pénalisants sur les trajectoires de sortie d'emploi chez les femmes que chez les hommes. À la suite d'arrêts maladie, la transition vers un éloignement du marché du travail est plus probable qu'un retour à l'emploi sans arrêt chez les femmes (odds ratio de 25,3 pour les femmes contre 15,3 pour les hommes). À l'inverse, les hommes ont une probabilité de persistance dans l'emploi associé

\section{Tableau 4}

\section{Modèles de transition, population féminine hors congés maternité (seuil à 60 jours)}

\begin{tabular}{|c|c|c|c|c|}
\hline & \multicolumn{4}{|c|}{ Situation d'emploi en $t$} \\
\hline Situation d'emploi en $t-1$ & $\begin{array}{c}(2) \\
\text { Emploi arrêts }<60 \mathrm{j}\end{array}$ & $\begin{array}{c}\text { (3) } \\
\text { Emploi arrêts }>60 \mathrm{j}\end{array}$ & $\begin{array}{c}\text { (4) } \\
\text { Chômage, activité réduite }\end{array}$ & $\begin{array}{c}(5) \\
\text { Non emploi }\end{array}$ \\
\hline \multicolumn{5}{|l|}{ Modèle multinomial } \\
\hline (1) Emploi sans arrêts & Réf. & Réf. & Réf. & Réf. \\
\hline (2) Emploi arrêts < 60j & $\begin{array}{c}2,92^{\star \star \star} \\
{[2,86 ; 3,00]}\end{array}$ & $\begin{array}{c}2,83^{\star \star \star} \\
{[2,74 ; 2,94]}\end{array}$ & $\begin{array}{c}1,60^{\star \star \star} \\
{[1,53 ; 1,67]}\end{array}$ & $\begin{array}{c}2,36^{\star \star \star} \\
{[2,19 ; 2,57]}\end{array}$ \\
\hline (3) Emploi arrêts $>60 j$ & $\begin{array}{c}3,16^{\star \star \star} \\
{[2,97 ; 3,36]}\end{array}$ & $\begin{array}{c}12,68^{\star \star \star} \\
{[11,95 ; 13,54]}\end{array}$ & $\begin{array}{c}4,85^{\star \star \star} \\
{[4,49 ; 5,29]}\end{array}$ & $\begin{array}{c}25,28^{\star \star \star} \\
{[23,05 ; 27,53]}\end{array}$ \\
\hline (4) Chômage/activité réduite & $\begin{array}{c}1,12^{\star \star \star} \\
{[1,07 ; 1,16]}\end{array}$ & $\begin{array}{c}1,12^{\star \star \star} \\
{[1,06 ; 1,19]}\end{array}$ & $\begin{array}{c}23,81^{\star \star \star} \\
{[23,08 ; 24,41]}\end{array}$ & $\begin{array}{c}32,79^{\star \star \star} \\
{[31,25 ; 34,65]}\end{array}$ \\
\hline (5) Non emploi & $\begin{array}{c}0,95 \\
{[0,87 ; 1,04]}\end{array}$ & $\begin{array}{c}1,15^{\star} \\
{[1,02 ; 1,28]}\end{array}$ & $\begin{array}{c}38,09^{\star \star \star} \\
{[36,64 ; 39,98]}\end{array}$ & $\begin{array}{c}464,05^{\star \star \star} \\
{[440,31 ; 493,21]}\end{array}$ \\
\hline \multicolumn{5}{|l|}{ Modèle à effets fixes } \\
\hline (1) Emploi sans arrêts & Réf. & Réf. & Réf. & Réf. \\
\hline (2) Emploi arrêts < 60j & $\begin{array}{c}1,16^{\star \star \star} \\
{[1,11 ; 1,22]}\end{array}$ & $\begin{array}{c}1,57^{\star \star \star} \\
{[1,40 ; 1,73]}\end{array}$ & $\begin{array}{c}1,17^{\star \star \star} \\
{[1,06 ; 1,31]}\end{array}$ & $\begin{array}{c}1,23^{\star \star} \\
{[1,02 ; 1,51]}\end{array}$ \\
\hline (3) Emploi arrêts > 60j & $\begin{array}{c}1,51^{\star \star \star} \\
{[1,34 ; 1,68]}\end{array}$ & $\begin{array}{c}5,99^{\star \star \star} \\
{[5,00 ; 7,24]}\end{array}$ & $\begin{array}{c}3,53^{\star \star \star} \\
{[2,86 ; 4,35]}\end{array}$ & $\begin{array}{c}9,39^{\star \star *} \\
{[7,10 ; 12,30]}\end{array}$ \\
\hline (4) Chômage/activité réduite & $\begin{array}{c}0,73^{\star \star \star} \\
{[0,66 ; 0,79]}\end{array}$ & $\begin{array}{c}0,88 \\
{[0,71 ; 1,08]}\end{array}$ & $\begin{array}{c}7,24^{\star \star \star} \\
{[6,62 ; 7,92]}\end{array}$ & $\begin{array}{c}19,30^{\star \star \star} \\
{[16,95 ; 22,20]}\end{array}$ \\
\hline (5) Non emploi & $\begin{array}{c}0,49^{\star \star \star} \\
{[0,40 ; 0,58]}\end{array}$ & $\begin{array}{c}0,90 \\
{[0,64 ; 1,26]}\end{array}$ & $\begin{array}{c}18,36^{\star \star \star} \\
{[16,28 ; 20,91]}\end{array}$ & $\begin{array}{c}206,44^{\star \star \star} \\
{[170,72 ; 249,64]}\end{array}$ \\
\hline
\end{tabular}

Lecture : dans le modèle à effets fixes, une femme ayant des arrêts maladie longs ou fréquents, par rapport à une femme en emploi sans arrêts, a 3,53 fois plus de chances d'être au chômage ou en activité réduite l'année suivante plutôt qu'en emploi sans arrêts au seuil de $0,1 \%$. Trois seuils de significativité sont calculés : ${ }^{*}<0,05 ;{ }^{* *}<0,01 ;{ }^{* * *}<0,001$. Les odds ratios estimés à partir du modèle naïf sont contrôlés de l'hétérogénéité observée (ALD, dépense totale de soins, âge en 2005, âge d'entrée sur le marché du travail, bénéficiaire CMU-C en $\mathrm{t}-1$, secteurs d'activité en $\mathrm{t}-1$ et indicatrice temporelle de transition).

Champ : modèle multinomial : femmes âgées de 25 à 55 ans n'ayant pas été en congé maternité entre 2005 et 2008 ( $n=357762$, données empilées) ; modèle à effets fixes : femmes âgées de 25 à 55 ans entre 2005 et 2008 n'ayant pas été en congé maternité " movers " entre 2006 et 2007 ( $n=37$ 374).

Source : Hygie (2005-2008). 
à des arrêts longs (état 3 ) plus élevée que les femmes (dépendance d'état dans l'état 3 associée à un odds ratio de 22,7 contre 12,7 chez les femmes) (tableaux 3 et 4). Ces résultats suggèrent qu'une détérioration de l'état de santé est plus souvent associée à un maintien en emploi avec des arrêts de travail chez les hommes alors qu'elle conduit davantage à une sortie d'activité chez les femmes.

Ces différences entre hommes et femmes peuvent être liées à des caractéristiques inobservées expliquant la plus grande élasticité de l'offre de travail des femmes face aux interruptions de carrières pour maladie. Les femmes, tout d'abord, ne disposent pas de la même protection sur le marché du travail. Au sein de la population salariée, elles occupent plus souvent des emplois à temps partiel que les hommes ${ }^{11}$. L'offre de travail des femmes est sensible au statut marital, au statut du conjoint et à la présence d'enfants (Edon et Kamionka, 2007; Pailhé et Solaz, 2012). On peut supposer qu'une dégradation de l'état de santé va davantage contraindre l'offre de travail des femmes que celle des hommes. Elle va modifier l'arbitrage travail/loisir voire les préférences entre production domestique et production marchande. Chez les femmes, les coûts d'opportunité de choisir la production domestique plutôt que la participation au marché du travail sont plus faibles en raison de leurs salaires moins élevés (Cahuc et Zylberberg, 2004) et l'inactivité est perçue de manière moins illégitime que pour les hommes (Bouffartigue, 2010). Enfin, il est possible que ce résultat soit en partie expliqué par la nature des maladies ou déficiences fonctionnelles auxquelles les hommes et les femmes font face (les femmes déclarent par exemple plus de limitations fonctionnelles motrices ou d'épisodes dépressifs (Célant et al., 2014)).

La correction de l'hétérogénéité fixe dans le temps annule ces différences entre hommes et femmes. Les risques de transitions vers le chômage et l'inactivité après des arrêts longs subsistent pour les hommes et les femmes - mais leur ampleur est comparable entre les deux groupes (avec des odds ratios de l'ordre de 3,5 pour le chômage et 9 pour l'inactivité). La probabilité de persistance dans de longs épisodes de maladie en emploi est également comparable chez les hommes et les femmes.

Il faut cependant également noter qu'une autre différence entre hommes et femmes ne subsiste pas après contrôle de l'hétérogénéité fixe dans le temps - à savoir la tendance des hommes à se retrouver un peu plus en emploi avec des arrêts longs après un épisode d'inactivité. Il est possible que le risque de dégradation de l'état de santé concomitant à la reprise d'emploi soit plus élevé chez les hommes en raison d'un effet de structure (le statut d'inactivité chez les hommes est plus souvent associé à des problèmes de santé que chez les femmes).

\section{Spécificités des congés maternité}

Dans la population féminine, considérer l'ensemble des arrêts pour raison de santé (congés maternité compris) réduit les risques associés aux épisodes d'emploi avec arrêts longs. Des arrêts de type congés maternité conduisent à des trajectoires professionnelles moins dégradées que des arrêts longs dus à la maladie - notamment parce que les congés maternité sont le plus souvent suivis d'un retour à l'emploi continu et sont plus rarement suivis d'arrêts maladie (graphique 2). Cet effet s'atténue cependant fortement une fois prise en compte l'hétérogénéité inobservée. Dans le modèle à effets fixes, l'odds ratio associé à une sortie vers le chômage pour les femmes issues de l'état 3 est de 3,2 en population générale (versus 3,5 hors congés maternité) (tableau 4 et annexe 3A). Les transitions vers le non-emploi sont aussi plus probables pour la population hors congés maternité $(9,4)$ par rapport à la population générale $(8,8)$.

Afin de s'assurer que les risques de trajectoires dégradées chez les femmes suite à des congés maladie longs comparés à ceux des femmes ayant eu des congés maternité ne sont pas seulement dus à l'âge plus élevé des premières, les estimations sont menées sur des sous-groupes homogènes (30-35 ans, 35-40 ans) $)^{12}$. Les résultats invitent à nuancer l'effet relativement moins pénalisant des congés maternités, car ils font apparaître certains risques spécifiques à chaque âge. Ainsi, l'inclusion des congés maternités dans l'échantillon féminin accroît le risque d'inactivité chez les 30-35 ans et accentue le risque de chômage chez les 35-40 ans.

\section{Des risques anticipés par les salariés ?}

Les résultats du modèle à effets fixes soulignent, par ailleurs, les risques anticipés par des personnes sortant d'épisodes de fragilité : en

11. D'après l'Insee, en 2012, 30,2 \% des femmes en emploi travaillent à temps partiel contre $6,9 \%$ des hommes.

12. Résultats non présentés, mais disponibles sur demande auprès des auteurs. 
effet, les individus retournant à l'emploi après une période de chômage, d'activité réduite ou de non emploi (états 4 et 5) ont une probabilité d'avoir des arrêts courts inférieure à ceux qui étaient initialement en emploi sans arrêts (état 1) (tableaux 3 et 4).

Ce résultat semble étayer l'hypothèse d'un lien causal entre insécurité de l'emploi et comportements de recours aux arrêts de travail et pourrait traduire l'existence d'un présentéisme accru parmi les salariés nouvellement embauchés. Plusieurs travaux montrent en effet, qu'en dépit d'une santé altérée, des salariés en situation de précarité face à l'emploi, avec un risque de chômage élevé, hésitent à recourir à des arrêts de travail (Hansen et Andersen, 2009). Il ne s'agit là que d'une hypothèse : les conditions d'indemnisation peuvent partiellement expliquer ce moindre recours aux arrêts maladie, puisqu'il faut justifier d'une durée de cotisation minimale pour bénéficier des indemnités journalières versées par l'assurance maladie. De plus, la reprise d'un emploi pourrait être associée à une amélioration de l'état de santé perçu.

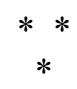

À partir d'un indicateur d'insertion professionnelle construit en cinq états (de l'emploi à des situations d'éloignement voire d'exclusion du marché du travail), cette étude met en évidence l'effet des arrêts maladie de courte et longue durée sur les trajectoires professionnelles l'année suivante.

Les résultats confirment la présence d'une dépendance d'état très marquée sur le marché du travail français, d'autant plus forte que l'éloignement du marché du travail est important. Ce constat s'applique également aux personnes exposées à des incapacités de travail dues à la santé. Ils mettent en évidence un risque de trajectoires dégradées plus élevé pour les salariés ayant eu des arrêts maladie que pour les salariés majoritairement en emploi. Ces effets subsistent une fois que l'on contrôle de l'hétérogénéité observée et inobservée fixe dans le temps, ce qui signifie qu'ils ne sont pas uniquement attribuables à des effets de composition au sein de ces états (facteurs associés à la fois à une mauvaise santé et une fragilité sur le marché du travail). De plus, les résultats suggèrent que les écarts de trajectoires consécutives à des arrêts maladie entre hommes et femmes s'expliquent davantage par des effets de ségrégation sur le marché du travail ou des caractéristiques propres à chaque sexe (notamment les préférences) que par un effet proprement discriminant de la maladie. Il est probable que les transitions dégradées de personnes ayant des incapacités reflètent en partie une détérioration de leur santé. Toutefois, le risque accru de chômage et d'inactivité consécutif à des épisodes de maladie reste problématique indépendamment de la dégradation de la santé qui peut l'accompagner. En revanche, les résultats de cette étude ne visent pas à saisir l'impact global de l'état de santé sur la trajectoire. Certains événements de santé comme les accidents du travail, les maladies professionnelles, et les invalidités permanentes, ne sont pas considérés dans le champ de l'analyse. Il est donc probable que l'effet de la santé sur les trajectoires professionnelles soit plus élevé que l'effet des arrêts maladie mesuré dans cet article.

Par ailleurs, l'effet des arrêts maladie sur le marché du travail est mesuré à travers le statut dans l'emploi ou les dispositifs d'indemnisation, sans qu'il ne soit possible de trancher sur le rôle respectif de l'offre ou de la demande de travail sur ces trajectoires. Plus modestement, ces résultats permettent de mettre en évidence que l'effet négatif de la maladie ne se manifeste non pas uniquement par des interruptions plus ou moins longues de travail, mais également par un risque accru de transition et de persistance dans le chômage et l'inactivité.

Si contrairement à Blasco et Givord (2010), cette étude ne modélise pas à proprement parler la durée des arrêts maladie, elle intègre cependant une mesure de durée cumulée combinant occurrence et durée des arrêts maladie dans les états d'emploi (de 1 jour à un an d'arrêt). Notons que cette durée maximale est suffisante étant donné qu'en dehors de cas exceptionnels (affections de longue durée), les salariés en arrêts maladie durant plus d'un an ne peuvent continuer à être indemnisés qu'au titre de l'invalidité. Les résultats permettent de conclure que la durée d'arrêts maladie est déterminante sur la trajectoire. Sans disposer d'une mesure fine de gravité de la santé des personnes en arrêt maladie, la distinction entre arrêts courts et arrêts longs met en évidence des trajectoires différenciées selon la durée de l'incapacité. L'examen du seuil à partir duquel les personnes ayant des arrêts maladie ont un risque significativement accru de trajectoires dégradées souligne que, pour les hommes et les femmes, les arrêts maladie longs sont plus pénalisants sur les trajectoires que les arrêts de moins de 30 jours. 
Les résultats établissent aussi des effets causaux inverses ; les personnes ayant eu des situations d'emploi défavorables sont moins susceptibles d'avoir recours à des arrêts courts lorsqu'elles reprennent un emploi ce qui peut suggérer un effet disciplinant du chômage, au risque d'accroître le présentéisme des précaires. On ne peut toutefois pas totalement exclure que le retour à l'emploi s'accompagne temporairement d'une amélioration réelle ou perçue de l'état de santé qui pourrait expliquer en partie un moindre recours aux arrêts de travail. Enfin, il est probable que les données administratives sous-estiment les arrêts maladie des anciens chômeurs de longue durée et des inactifs (état 5) qui ne disposent pas nécessairement de droits suffisants pour prétendre à une indemnisation de leurs arrêts maladie ${ }^{13}$.

Les risques anticipés par les individus de pénalités associés aux arrêts maladie semblent toutefois bien réels. Les résultats suggèrent qu'un arrêt maladie long peut constituer un obstacle à une réinsertion sociale rapide et stable. On sait que le secteur privé assure une moins bonne

13. L'indemnisation des arrêts maladie est soumise à une durée de cotisation minimale de 200 heures au cours des trois mois précédant l'arrêt de travail ou d'un salaire au moins égal à 1015 fois le montant du Smic horaire au cours des six mois précédant l'arrêt de travail.

14. La fonction publique assure un maintien dans l'emploi (ou un retour à l'emploi) plus fréquent que le secteur privé notamment, grâce à certaines dispositions (garantie de l'emploi, obligation de reclassement, ...).

15. À ce titre, dans le troisième Plan cancer 2014-2019, il est indiqué la nécessité de "diminuer l'impact du cancer sur la vie personnelle" afin notamment d'éviter des phénomènes de "double peine » (maladie et exclusion du marché du travail). En outre, depuis la circulaire du 4 septembre 2012, tout projet de loi doit désormais inclure des dispositions spécifiques aux personnes handicapées, notamment en matière d'insertion professionnelle.

16. Ce type de congés non rémunérés n'est pas considéré dans notre analyse. II s'adresse aux salariés, justifiant d'une certaine ancienneté dans l'entreprise, qui souhaitent cesser leur activité pour s'occuper d'un proche présentant un handicap ou une perte d'autonomie. protection des salariés face à un choc de type handicap ou maladies chroniques que les salariés du public ${ }^{14}$. Ces résultats renforcent donc la nécessité d'assurer une meilleure réintégration des personnes malades issus du secteur privé ; principe réaffirmé par la mise en place récente du troisième Plan Cancer ou des dernières dispositions à l'encontre des personnes handicapées ${ }^{15}$.

Cette première étude française sur l'impact des arrêts maladie appelle naturellement des prolongements. La relation entre les arrêts maladie et les trajectoires varie certainement selon les populations. Le cas des personnes en fin de carrière, par exemple, serait particulièrement intéressant à étudier dans la mesure où les arrêts maladie pourraient être utilisés comme dispositifs de cessation anticipée d'activité. Cette étude nécessiterait d'isoler les départs en retraite choisis des trajectoires d'inactivité davantage subies.

Les personnes confrontées à des charges familiales particulières pourraient également être à la fois plus exposées aux arrêts maladie et à des trajectoires fragilisées. C'est le cas de la prise en charge d'un parent dépendant, d'un conjoint malade ou d'un enfant malade qui autorise un congé de soutien familial obtenu de droit sous certaines conditions ${ }^{16}$. L'analyse de ces trajectoires d'emploi et de santé en lien avec les événements familiaux n'est pas encore possible avec les données administratives existantes. Enfin, le prolongement naturel de cette étude serait d'analyser les pénalités associées aux arrêts maladie des salariés se maintenant en emploi, en suivant les caractéristiques de l'emploi occupé à la suite de ces arrêts, et d'analyser la réversibilité des trajectoires en analysant les effets des arrêts maladie à plus long terme. Ces études pourraient bénéficier de l'allongement progressif du panel Hygie.

\section{BIBLIOGRAPHIE}

Akashi-Ronquest N., Carrillo P., Dembling B. et Stern S. (2011), « Measuring the biases in self-reported disability status: evidence from aggregate data ", Applied Economics Letters, vol. $18, \mathrm{n}^{\circ} 11$, pp. 1053-1060.

Barmby T., Sessions J. et Treble J. (1994), "Absenteeism, efficiency wages and shirking ",
The Scandinavian Journal of Economics, vol. 96, $\mathrm{n}^{\circ} 4$, pp. 561-566.

Barnay T et Briard K, (2009) « Carrière incomplète et départ en retraite: une estimation de l'incidence de l'état de santé à partir de données individuelles », Revue économique, Vol. 60, $\mathrm{n}^{\circ} 2$, pp. 345-364. 
Barnay T. (2005), «Santé déclarée et cessation d'activité ", Revue française d'économie, vol. 20, $\mathrm{n}^{\circ} 2$, pp. 73-106.

Beck S. et Kamionka T. (2012), « Mobilités, inégalités et trajectoires professionnelles », Revue économique, vol. 63, n 3, pp. 453-464.

Blasco S. et Givord P. (2010), « Les trajectoires professionnelles en début de vie active: quel impact des contrats temporaires? », Économie et Statistique, $\mathrm{n}^{\circ}$ 431-432, pp. 73-93.

Bouffartigue P. (2010), « La perception des liens travail/santé », Revue française de sociologie, vol. $51, \mathrm{n}^{\circ} 2$, pp. 247-280.

Bound J. (1991), « Self-Reported Versus Objective Measures of Health in Retirement Models », Journal of Human Resources, vol. 26, $\mathrm{n}^{\circ}$ 1, pp. 106-138.

Bound J., Schoenbaum M., Stinebrickner T. R. et Waidmann T. (1999), « The dynamic effects of health on the labor force transitions of older workers ", Labour Economics, vol. 6, $\mathrm{n}^{\circ} 2$, pp. 179-202.

Bouvier G. et Jugnot S. (2014), « Les personnes ayant des problèmes de santé ou de handicap sont plus nombreuses que les autres à faire part de comportements stigmatisants », Économie et Statistique, $\mathrm{n}^{\circ}$ 464-465-466, pp. 189-213.

Brodaty T. (2008), « Introduire de l'hétérogénéité inobservée dans la mobilité des salaires », Revue économique, vol. 59, $\mathrm{n}^{\circ}$ 3, pp. 621-629.

Cahuc P. A. et Zylberberg A. A. (2004), Labor economics, MIT press.

Chaupain-Guillot S., Guillot O. et Wolff F.-C. (2007), «Les absences au travail : une analyse à partir des données françaises du Panel européen des ménages ; suivi d'un commentaire de François-Charles Wolff », Économie et statistique, vol. $408, \mathrm{n}^{\circ} 1$, pp. 45-80.

Contoyannis P. et Rice N. (2001), « The impact of health on wages: evidence from the British Household Panel Survey », Empirical Economics, vol. $26, \mathrm{n}^{\circ} 4$, pp. 599-622.

Célant N., Guillaume S. et Rochereau T. (2014), "Enquête sur la santé et la protection sociale $2012 »$, Rapport de l'Irdes, n 556, 302 p.

Edon C. et Kamionka T. (2007), « Modélisation dynamique de la participation au marché du tra- vail des femmes en couple », Annals of Economics and Statistics / Annales d'Économie et de Statistique, $\mathrm{n}^{\circ} 86$, pp. 77-108.

Erhel C. et Guergoat-Larivière M. (2013), « La mobilité de la main-d'œuvre en Europe », Revue économique, vol. 64, $\mathrm{n}^{\circ} 2$, pp. 309-343.

Etilé F. et Milcent C. (2006), «Income-related reporting heterogeneity in self-assessed health: evidence from France ", Health economics, vol. $15, \mathrm{n}^{\circ} 9$, pp. $965-981$.

Givord P. et Wilner L. (2009), «Short-term contracts: trap or stepping stone toward stable employment? », Document de travail Insee-Dese.

Haan P. et Myck M. (2009), «Dynamics of health and labor market risks », Journal of Health Economics, vol. 28, n 6 , pp. 1116-1125.

Hansen C. D. et Andersen J. H. (2009), « Sick at work-a risk factor for long-term sickness absence at a later date? ", Journal of epidemiology and community health, vol. 63, $\mathrm{n}^{\circ} 5$, pp. 397-402.

Hansen J. (2000), « The effect of work absence on wages and wage gaps in Sweden », Journal of Population Economics, vol. 13, n 1, pp. 45-55.

Havet N. (2006), «L'insertion professionnelle des jeunes et mesures publiques: des trajectoires différenciées entre hommes et femmes ", Annales d'Économie et de Statistique, pp. 225-251.

Heckman J. J. (1981), « Heterogeneity and state dependence », dans Studies in labor markets, University of Chicago Press, pp. 91-140.

Hesselius P. (2007), « Does sickness absence increase the risk of unemployment? », The Journal of Socio-Economics, vol. 36, $\mathrm{n}^{\circ} 2$, pp. 288-310.

Honoré B. E. et Kyriazidou E. (2000), « Panel data discrete choice models with lagged dependent variables »,Econometrica, vol. 68, n 4,pp. 839-874.

Ichino A et Riphahn T. T (2005), « The effect of employment protection on work effort: absenteeism during and after probation », Journal of the European Economic Association, vol. 3, $\mathrm{n}^{\circ} 1$, pp. 120-143.

Jusot F., Khalt M., Rochereau T. et Sermet C. (2008), «Job loss from ill-health, smoking and obesity: concurrent evidence for direct and indirect selection », Journal of epidemiology and community health, vol. $62, \mathrm{n}^{\circ} 4$, pp. 332-337. 
Le Garrec M. A. et Bouvet M. (2013), « Les Comptes nationaux de la santé en 2012 », Document de travail, Drees, $\mathrm{n}^{\circ} 851$, septembre.

Lê F. et Reynaud D. (2007), «Les indemnités journalières », Études et résultats, Drees, n 592.

Lindeboom M. (2006), « Health and work of older workers ", The Elgar companion to health economics, pp. 26.

Lumsdaine R. L. et Mitchell O. S. (1999), «New developments in the economic analysis of retirement », Handbook of labor economics, vol. 3, pp. 3261-3307.

Magnac T. (2000), «Subsidised training and youth employment: distinguishing unobserved heterogeneity from state dependence in labour market histories », The Economic Journal, vol. $110, \mathrm{n}^{\circ} 466$, pp. 805-837.

Markussen S. (2012), «The individual cost of sick leave », Journal of Population Economics, vol. $25, \mathrm{n}^{\circ} 4$, pp. 1287-1306.

Mesrine A. (2000), «La surmortalité des chômeurs: un effet catalyseur du chômage? », Économie et statistique, vol. 334, $\mathrm{n}^{\circ} 1$, pp. 33-48.

Meurs D. et Ponthieux S. (2006), «L'écart des salaires entre les femmes et les hommes peut-il encore baisser?», Économie et statistique, vol. $398, \mathrm{n}^{\circ} 1$, pp. 99-129.

OCDE (2010), Sickness, Disability and Work: Breaking the Barriers; A Synthesis of Findings Across Oecd Countries.

Pailhé A. et Solaz A. (2012), «Durée et conditions de retour à l'emploi des mères après une naissance », Retraite et société, vol. 63, n² 2, pp. 51-77.

Ross C. E. et Mirowsky J. (1995), « Does employment affect health? », Journal of Health and social Behavior, pp. 230-243.

Salm M. (2009), « Does job loss cause ill health? », Health Economics, vol. 18, n 9, pp. 1075-1089.

Shmueli A. (2003), « Socio-economic and demographic variation in health and in its measures: the issue of reporting heterogeneity », Social science \& medicine, vol. 57, $\mathrm{n}^{\circ} 1$, pp. 125-134.

Tessier P. et Wolff F.-C. (2005), « Offre de travail et santé en France », Économie \& prévision, n ${ }^{\circ}$, pp. 17-41.

Wooldridge J. M. (2005), « Simple solutions to the initial conditions problem in dynamic, nonlinear panel data models with unobserved heterogeneity », Journal of applied econometrics, vol. 20 , $\mathrm{n}^{\circ} 1$, pp. 39-54. 
ANNEXE 1

ÉCHANTILLON: RÔLE DES FILTRES APPLIQUÉS

\begin{tabular}{|l|c|}
\hline \multicolumn{1}{|c|}{ Filtres } & Effectifs \\
\hline Échantillon initial & 520838 \\
Suppression des moins de 25 ans en 2005 & 481426 \\
Suppression des plus de 55 ans en 2008 & 339659 \\
Suppression des individus décédés sur la période 2005-2008 & 339568 \\
Suppression des individus cotisants à un autre régime que le régime général & 325932 \\
Suppression des individus ayant validé des PA rentes & 319020 \\
Suppression des individus ayant eu des ATMP & 285008 \\
Cylindrage (individus présents de 2005 à 2008) & 247779 \\
\hline
\end{tabular}

Lecture : la suppression des moins de 25 ans en 2005 réduit la taille de l'échantillon de 520838 à 481426 individus. Après application de l'ensemble des filtres et le cylindrage, l'échantillon d'analyse contient 247779 individus.

Champ : échantillon de salariés affiliés au Régime général de retraite.

Source : Hygie (2005-2008). 


\section{TRANSITIONS ANNUELLES BRUTES DE LA POPULATION DES «MOVERS »}

\section{A. Hommes}

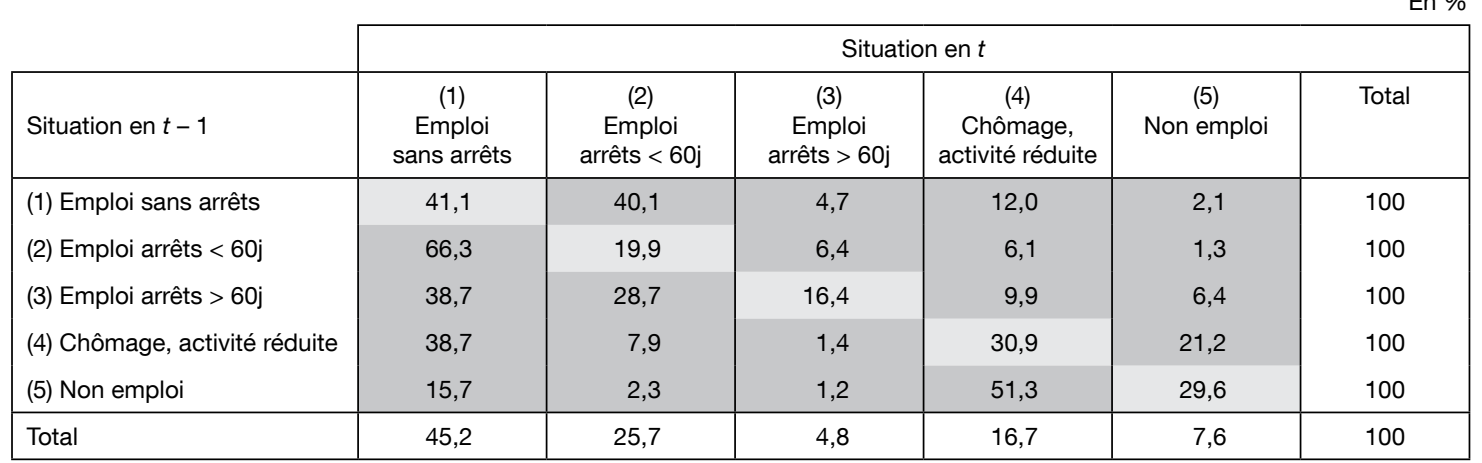

Lecture : parmi les hommes "movers » en emploi sans arrêt maladie en t-1 (état 1), 12 \% ont transité vers le chômage ou l'activité réduite en $\mathrm{t}$ (état 4).

Champ : salariés du privé âgés de 25 à 55 ans, " movers » entre 2006 et 2007 ( $n=37567)$

Source : Hygie (2005-2008).

\section{B. Femmes (hors congés maternité)}

En \%

\begin{tabular}{|c|c|c|c|c|c|c|}
\hline \multirow[b]{2}{*}{ Situation en $t-1$} & \multicolumn{6}{|c|}{ Situation en $t$} \\
\hline & $\begin{array}{c}\text { (1) } \\
\text { Emploi } \\
\text { sans arrêts }\end{array}$ & $\begin{array}{c}(2) \\
\text { Emploi } \\
\text { arrêts < 60j }\end{array}$ & $\begin{array}{c}(3) \\
\text { Emploi } \\
\text { arrêts > 60j }\end{array}$ & $\begin{array}{c}\text { (4) } \\
\text { Chômage, } \\
\text { activité réduite }\end{array}$ & $\begin{array}{c}(5) \\
\text { Non emploi }\end{array}$ & Total \\
\hline (1) Emploi sans arrêts & 41,1 & 38,5 & 5,2 & 13,0 & 2,2 & 100 \\
\hline (2) Emploi arrêts < 60j & 61,1 & 22,4 & 7,8 & 7,3 & 1,6 & 100 \\
\hline (3) Emploi arrêts > 60j & 33,1 & 27,7 & 17,4 & 11,8 & 10,1 & 100 \\
\hline (4) Chômage, activité réduite & 38,0 & 9,6 & 2,0 & 31,1 & 19,4 & 100 \\
\hline (5) Non emploi & 14,5 & 3,0 & 0,9 & 48,6 & 33,0 & 100 \\
\hline Total & 41,6 & 23,9 & 5,1 & 19,7 & 9,7 & 100 \\
\hline
\end{tabular}

Lecture : parmi les femmes " movers » (hors congés maternité) en emploi sans arrêt maladie en $\mathrm{t}-1$ (état 1), $13 \%$ ont transité vers chômage ou l'activité réduite en $\mathrm{t}$ (état 4).

Champ : salariées du privé âgées de 25 à 55 ans, « movers » entre 2006 et 2007, n'ayant pas eu de congé maternité en t - 1 ( $n=37374$ ). Source : Hygie (2005-2008).

\section{Femmes (congés maternité compris)}

En \%

\begin{tabular}{|c|c|c|c|c|c|c|}
\hline \multirow[b]{2}{*}{ Situation en $t-1$} & \multicolumn{6}{|c|}{ Situation en $t$} \\
\hline & $\begin{array}{c}\text { (1) } \\
\text { Emploi } \\
\text { sans arrêts }\end{array}$ & $\begin{array}{c}(2) \\
\text { Emploi } \\
\text { arrêts < 60j }\end{array}$ & $\begin{array}{c}(3) \\
\text { Emploi } \\
\text { arrêts > 60j }\end{array}$ & $\begin{array}{c}\text { (4) } \\
\text { Chômage, } \\
\text { activité réduite }\end{array}$ & $\begin{array}{c}(5) \\
\text { Non emploi }\end{array}$ & Total \\
\hline (1) Emploi sans arrêts & 38,8 & 34,9 & 12,2 & 11,9 & 2,3 & 100 \\
\hline (2) Emploi arrêts < 60j & 56,2 & 20,4 & 14,7 & 6,9 & 2,0 & 100 \\
\hline (3) Emploi arrêts > 60j & 42,3 & 20,8 & 11,7 & 12,2 & 13,0 & 100 \\
\hline (4) Chômage, activité réduite & 36,2 & 9,2 & 3,7 & 29,6 & 21,3 & 100 \\
\hline (5) Non emploi & 14,4 & 3,0 & 1,5 & 46,4 & 34,8 & 100 \\
\hline Total & 39,6 & 21,6 & 9,7 & 18,3 & 10,7 & 100 \\
\hline
\end{tabular}

Lecture : parmi les femmes " movers » (hors congés maternité) en emploi sans arrêt maladie en $\mathrm{t}-1$ (état 1), 11,9\% ont transité vers chômage ou l'activité réduite en $\mathrm{t}$ (état 4).

Champ : salariées du privé âgées de 25 à 55 ans, " movers » entre 2006 et 2007 ( $n=49$ 547).

Source: Hygie (2005-2008). 


\section{MODÈLES DE TRANSITION}

A. Population féminine congés maternité compris (seuil à 60 jours)

\begin{tabular}{|c|c|c|c|c|}
\hline \multirow[b]{2}{*}{ Situation d'emploi en $t-1$} & \multicolumn{4}{|c|}{ Situation d'emploi en $t$} \\
\hline & $\begin{array}{c}(2) \\
\text { Emploi arrêts < 60j }\end{array}$ & $\begin{array}{c}(3) \\
\text { Emploi arrêts > 60j }\end{array}$ & $\begin{array}{l}\text { (4) } \\
\text { Chômage, activité réduite }\end{array}$ & $\begin{array}{c}(5) \\
\text { Non emploi }\end{array}$ \\
\hline \multicolumn{5}{|l|}{ Modèle multinomial } \\
\hline (1) Emploi sans arrêts & Réf. & Réf. & Réf. & Réf. \\
\hline (2) Emploi arrêts < 60j & $\begin{array}{c}2,92^{\star \star \star} \\
{[2,86 ; 3,00]}\end{array}$ & $\begin{array}{c}2,83^{\star \star \star} \\
{[2,73 ; 2,93]}\end{array}$ & $\begin{array}{c}1,600^{\star \star \star} \\
{[1,53 ; 1,68]}\end{array}$ & $\begin{array}{c}2,36^{\star \star \star} \\
{[2,19 ; 2,57]}\end{array}$ \\
\hline (3) Emploi arrêts > 60j & $\begin{array}{c}1,75^{\star \star \star} \\
{[1,68 ; 1,82]}\end{array}$ & $\begin{array}{c}3,03^{\star \star \star} \\
{[2,90 ; 3,20]}\end{array}$ & $\begin{array}{c}3,19^{\star \star \star} \\
{[3,02 ; 3,36]}\end{array}$ & $\begin{array}{c}17,46^{\star \star \star} \\
{[16,44 ; 18,69]}\end{array}$ \\
\hline (4) Chômage/activité réduite & $\begin{array}{c}1,12^{\star \star \star} \\
{[1,07 ; 1,16]}\end{array}$ & $\begin{array}{c}1,12^{\star \star \star} \\
{[1,05 ; 1,18]}\end{array}$ & $\begin{array}{c}23,81^{\star \star \star} \\
{[23,20 ; 24,54]}\end{array}$ & $\begin{array}{c}34,12^{\star \star \star} \\
{[32,54 ; 36,05]}\end{array}$ \\
\hline (5) Non emploi & $\begin{array}{c}0,96 \\
{[0,88 ; 1,04]}\end{array}$ & $\begin{array}{c}1,12^{\star \star} \\
{[1,00 ; 1,25]}\end{array}$ & $\begin{array}{c}37,71^{\star \star \star} \\
{[36,14 ; 39,37]}\end{array}$ & $\begin{array}{c}464,05^{\star \star \star} \\
{[440,72 ; 492,95]}\end{array}$ \\
\hline \multicolumn{5}{|l|}{ Modèle à effets fixes } \\
\hline (1) Emploi sans arrêts & Réf. & Réf. & Réf. & Réf. \\
\hline (2) Emploi arrêts < 60j & $\begin{array}{c}1,199^{\star \star \star} \\
{[1,13 ; 1,25]}\end{array}$ & $\begin{array}{c}1,72^{\star \star \star} \\
{[1,60 ; 1,82]}\end{array}$ & $\begin{array}{c}1,21^{\star \star *} \\
{[1,11 ; 1,34]}\end{array}$ & $\begin{array}{c}1,54^{\star \star \star} \\
{[1,30 ; 1,80]}\end{array}$ \\
\hline (3) Emploi arrêts > 60j & $\begin{array}{c}1,09^{\star \star} \\
{[1,02 ; 1,17]}\end{array}$ & $\begin{array}{c}1,95^{\star \star \star} \\
{[1,77 ; 2,16]}\end{array}$ & $\begin{array}{c}3,19^{\star \star \star} \\
{[2,83 ; 3,60]}\end{array}$ & $\begin{array}{c}8,76^{\star \star \star} \\
{[7,39 ; 10,28]}\end{array}$ \\
\hline (4) Chômage/activité réduite & $\begin{array}{c}0,72^{\star \star \star} \\
{[0,66 ; 0,78]}\end{array}$ & $\begin{array}{c}0,70^{\star \star \star} \\
{[0,62 ; 0,79]}\end{array}$ & $\begin{array}{c}7,17^{\star \star \star} \\
{[6,62 ; 7,85]}\end{array}$ & $\begin{array}{c}19,11^{\star \star \star} \\
{[16,95 ; 21,76]}\end{array}$ \\
\hline (5) Non emploi & $\begin{array}{c}0,51^{\star \star \star} \\
{[0,43 ; 0,60]}\end{array}$ & $\begin{array}{c}0,62^{\star \star \star} \\
{[0,50 ; 0,77]}\end{array}$ & $\begin{array}{c}17,64^{\star \star \star} \\
{[15,80 ; 16,69]}\end{array}$ & $\begin{array}{c}198,34^{\star \star \star} \\
{[167,34 ; 235,10]}\end{array}$ \\
\hline
\end{tabular}

Lecture : dans le modèle à effets fixes, une femme ayant des arrêts maladie longs ou fréquents, par rapport à une femme en emploi sans arrêts, a 3,19 fois plus de chances d'être au chômage ou en activité réduite l'année suivante plutôt qu'en emploi sans arrêts au seuil de $0,1 \%$. Trois seuils de significativité sont calculés : ${ }^{\star}<0,05 ;{ }^{* *}<0,01 ;{ }^{* *}<0,001$. Les odds ratios estimés à partir du modèle naïf sont contrôlés de l'hétérogénéité observée (ALD, dépense totale de soins, âge en 2005, âge d'entrée sur le marché du travail, bénéficiaire CMU-C en $\mathrm{t}-1$, secteurs d'activité en $\mathrm{t}-1$ et indicatrice temporelle de transition).

Champ : modèle multinomial : femmes âgées de 25 à 55 ans entre 2005 et 2008 ( $n=374$ 067, données empilées); modèle à effets fixes : femmes âgées de 25 à 55 ans entre 2005 et 2008 « movers » entre 2006 et 2007 ( $n=49547)$.

Source : Hygie (2005-2008). 
B. Population masculine (seuil à 30 jours)

\begin{tabular}{|c|c|c|c|c|}
\hline \multirow[b]{2}{*}{ Situation d'emploi en $t-1$} & \multicolumn{4}{|c|}{ Situation d'emploi en $t$} \\
\hline & $\begin{array}{c}\text { (2) } \\
\text { Emploi arrêts }<30 j\end{array}$ & $\begin{array}{c}\text { (3) } \\
\text { Emploi arrêts > 30j }\end{array}$ & $\begin{array}{c}(4) \\
\text { Chômage, activité réduite }\end{array}$ & $\begin{array}{c}\text { (5) } \\
\text { Non emploi }\end{array}$ \\
\hline \multicolumn{5}{|l|}{ Modèle multinomial } \\
\hline (1) Emploi sans arrêts & Réf. & Réf. & Réf. & Réf. \\
\hline (2) Emploi arrêts < 30j & $\begin{array}{c}3,10^{\star \star \star} \\
{[3,03 ; 3,19]}\end{array}$ & $\begin{array}{c}3,19^{\star \star \star} \\
{[3,04 ; 3,32]}\end{array}$ & $\begin{array}{c}1,77^{\star \star *} \\
{[1,69 ; 1,87]}\end{array}$ & $\begin{array}{c}1,84^{\star \star *} \\
{[1,65 ; 2,06]}\end{array}$ \\
\hline (3) Emploi arrêts > 30j & $\begin{array}{c}2,56^{\star \star \star} \\
{[2,43 ; 2,68]}\end{array}$ & $\begin{array}{c}9,21^{\star \star *} \\
{[8,69 ; 9,66]}\end{array}$ & $\begin{array}{c}3,53^{\star \star *} \\
{[3,30 ; 3,81]}\end{array}$ & $\begin{array}{c}8,67^{\star \star \star} \\
{[7,84 ; 9,62]}\end{array}$ \\
\hline (4) Chômage/activité réduite & $\begin{array}{c}1,17^{\star \star \star} \\
{[1,12 ; 1,24]}\end{array}$ & $\begin{array}{c}1,63^{\star \star \star} \\
{[1,50 ; 1,76]}\end{array}$ & $\begin{array}{c}30,27^{\star \star \star} \\
{[29,41 ; 31,27]}\end{array}$ & $\begin{array}{c}48,91^{\star \star \star} \\
{[46,24 ; 51,93]}\end{array}$ \\
\hline (5) Non emploi & $\begin{array}{c}0,85^{\star \star \star} \\
{[0,73 ; 0,99]}\end{array}$ & $\begin{array}{c}2,41^{\star \star \star} \\
{[2,04 ; 2,84]}\end{array}$ & $\begin{array}{c}54,05^{\star \star \star} \\
{[51,03 ; 57,20]}\end{array}$ & $\begin{array}{c}464,05^{\star \star \star} \\
{[431,51 ; 496,51]}\end{array}$ \\
\hline \multicolumn{5}{|l|}{ Modèle à effets fixes } \\
\hline (1) Emploi sans arrêts & Réf. & Réf. & Réf. & Réf. \\
\hline (2) Emploi arrêts < 30j & $\begin{array}{c}1,08^{\star \star} \\
{[1,03 ; 1,15]}\end{array}$ & $\begin{array}{c}1,27^{\star \star \star} \\
{[1,17 ; 1,38]}\end{array}$ & $\begin{array}{c}1,16^{\star \star \star} \\
{[1,04 ; 1,30]}\end{array}$ & $\begin{array}{c}1,19 \\
{[0,97 ; 1,46]}\end{array}$ \\
\hline (3) Emploi arrêts > 30j & $\begin{array}{c}1,05 \\
{[0,97 ; 1,15]}\end{array}$ & $\begin{array}{c}2,64^{\star \star *} \\
{[2,34 ; 2,97]}\end{array}$ & $\begin{array}{c}2,18^{\star \star \star} \\
{[1,86 ; 2,59]}\end{array}$ & $\begin{array}{c}4,76^{\star \star \star} \\
{[3,71 ; 6,11]}\end{array}$ \\
\hline (4) Chômage/activité réduite & $\begin{array}{c}0,72^{\star \star \star} \\
{[0,64 ; 0,80]}\end{array}$ & $\begin{array}{c}0,90 \\
{[0,76 ; 1,06]}\end{array}$ & $\begin{array}{c}7,10^{\star \star \star} \\
{[6,49 ; 7,77]}\end{array}$ & $\begin{array}{c}18,92^{\star \star \star} \\
{[16,61 ; 21,76]}\end{array}$ \\
\hline (5) Non emploi & $\begin{array}{c}0,58^{\star \star \star} \\
{[0,45 ; 0,75]}\end{array}$ & $\begin{array}{c}1,13 \\
{[0,81 ; 1,55]}\end{array}$ & $\begin{array}{c}17,46^{\star \star \star} \\
{[15,33 ; 20,09]}\end{array}$ & $\begin{array}{c}138,38^{\star \star \star} \\
{[113,30 ; 170,72]}\end{array}$ \\
\hline
\end{tabular}

Lecture : dans le modèle à effets fixes, un homme ayant des arrêts maladie longs ou fréquents, par rapport à un homme en emploi sans arrêts, a 3,53 fois plus de chances d'être au chômage ou en activité réduite l'année suivante plutôt qu'en emploi sans arrêts au seuil de $0,1 \%$. Trois seuils de significativité sont calculés : ${ }^{*}<0,05 ;{ }^{* *}<0,01 ;{ }^{* * *}<0,001$. Les odds ratios estimés à partir du modèle naïf sont contrôlés de l'hétérogénéité observée (ALD en $\mathrm{t}-1$, dépense totale de soins en $\mathrm{t}-1$, âge en 2005 , âge d'entrée sur le marché du travail, bénéficiaire CMU-C en $\mathrm{t}-1$, secteurs d'activité en $\mathrm{t}-1$ et indicatrice temporelle de transition).

Champ : modèle multinomial : hommes âgés de 25 à 55 ans ( $n=369$ 267, données empilées); modèle à effets fixes : hommes âgés de 25 à 55 ans « movers » entre 2006 et 2007 ( $n=38$ 178).

Source: Hygie (2005-2008).

\section{Population féminine hors congés maternité (seuil à 30 jours)}

odds ratios

\begin{tabular}{|c|c|c|c|c|}
\hline \multirow[b]{2}{*}{ Situation d'emploi en $t-1$} & \multicolumn{4}{|c|}{ Situation d'emploi en $t$} \\
\hline & $\begin{array}{c}(2) \\
\text { Emploi arrêts }<30 j\end{array}$ & $\begin{array}{c}(3) \\
\text { Emploi arrêts > 30j }\end{array}$ & $\begin{array}{c}(4) \\
\text { Chômage, activité réduite }\end{array}$ & $\begin{array}{c}(5) \\
\text { Non emploi }\end{array}$ \\
\hline \multicolumn{5}{|l|}{ Modèle multinomial } \\
\hline (1) Emploi sans arrêts & Réf. & Réf. & Réf. & Réf. \\
\hline (2) Emploi arrêts < 30j & $\begin{array}{c}2,89^{\star \star \star} \\
{[2,80 ; 2,96]}\end{array}$ & $\begin{array}{c}2,66^{\star \star \star} \\
{[2,58 ; 2,75]}\end{array}$ & $\begin{array}{c}1,49^{\star \star \star} \\
{[1,43 ; 1,57]}\end{array}$ & $\begin{array}{c}2,01^{\star \star \star} \\
{[1,84 ; 2,21]}\end{array}$ \\
\hline (3) Emploi arrêts $>30 j$ & $\begin{array}{c}2,56^{\star \star \star} \\
{[2,45 ; 2,69]}\end{array}$ & $\begin{array}{c}6,49^{\star \star \star} \\
{[6,23 ; 6,80]}\end{array}$ & $\begin{array}{c}3,35^{\star \star \star} \\
{[3,14 ; 3,56]}\end{array}$ & $\begin{array}{c}13,33^{\star \star \star} \\
{[12,32 ; 14,39]}\end{array}$ \\
\hline (4) Chômage/activité réduite & $\begin{array}{c}1,11^{\star \star \star} \\
{[1,06 ; 1,15]}\end{array}$ & $\begin{array}{c}1,14^{\star \star} \\
{[1,08 ; 1,19]}\end{array}$ & $\begin{array}{c}23,81^{\star \star \star} \\
{[23,13 ; 24,47]}\end{array}$ & $\begin{array}{c}32,46^{\star \star \star} \\
{[30,91 ; 34,26]}\end{array}$ \\
\hline (5) Non emploi & $\begin{array}{c}0,94 \\
{[0,86 ; 1,04]}\end{array}$ & $\begin{array}{c}1,16^{\star \star} \\
{[1,05 ; 1,28]}\end{array}$ & $\begin{array}{c}38,09^{\star \star \star} \\
{[36,58 ; 39,92]}\end{array}$ & $\begin{array}{c}464,05^{\star \star \star} \\
{[436,44 ; 488,72]}\end{array}$ \\
\hline \multicolumn{5}{|l|}{ Modèle à effets fixes } \\
\hline (1) Emploi sans arrêts & Réf. & Réf. & Réf. & Réf. \\
\hline (2) Emploi arrêts < 30j & $\begin{array}{c}1,12^{\star \star \star} \\
{[1,05 ; 1,18]}\end{array}$ & $\begin{array}{c}1,34^{\star \star \star} \\
{[1,23 ; 1,45]}\end{array}$ & $\begin{array}{c}1,11^{\star} \\
{[0,99 ; 1,23]}\end{array}$ & $\begin{array}{c}1,14 \\
{[0,92 ; 1,39]}\end{array}$ \\
\hline (3) Emploi arrêts > 30j & $\begin{array}{c}1,16^{\star \star \star} \\
{[1,07 ; 1,27]}\end{array}$ & $\begin{array}{c}2,51^{\star \star \star} \\
{[2,23 ; 2,83]}\end{array}$ & $\begin{array}{c}2,32^{\star \star \star} \\
{[1,97 ; 2,72]}\end{array}$ & $\begin{array}{c}5,00^{\star \star \star} \\
{[2,72 ; 6,30]}\end{array}$ \\
\hline (4) Chômage/activité réduite & $\begin{array}{c}0,74^{\star \star \star} \\
{[0,67 ; 0,82]}\end{array}$ & $\begin{array}{c}0,80^{\star \star \star} \\
{[0,69 ; 0,93]}\end{array}$ & $\begin{array}{c}7,24^{\star \star \star} \\
{[6,62 ; 7,92]}\end{array}$ & $\begin{array}{c}19,30^{\star \star \star} \\
{[16,78 ; 22,20]}\end{array}$ \\
\hline (5) Non emploi & $\begin{array}{c}0,49^{\star \star \star} \\
{[0,40 ; 0,59]}\end{array}$ & $\begin{array}{c}0,72 \\
{[0,55 ; 0,93]}\end{array}$ & $\begin{array}{c}18,36^{\star \star \star} \\
{[16,28 ; 20,70]}\end{array}$ & $\begin{array}{c}204,38^{\star \star \star} \\
{[169,02 ; 247,15]}\end{array}$ \\
\hline
\end{tabular}

Lecture : dans le modèle à effets fixes, une femme ayant des arrêts maladie longs ou fréquents, par rapport à une femme en emploi sans arrêts, a 2,4 fois plus de chances d'être au chômage ou en activité réduite l'année suivante plutôt qu'en emploi sans arrêts au seuil de $0,1 \%$. Trois seuils de significativité sont calculés : ${ }^{*}<0,05 ;{ }^{* *}<0,01 ;{ }^{* * *}<0,001$. Les odds ratios estimés à partir du modèle naïf sont contrôlés de l'hétérogénéité observée ( $A L D$, dépense totale de soins, âge en 2005, âge d'entrée sur le marché du travail, bénéficiaire CMU-C en $\mathrm{t}-1$, secteurs d'activité en $\mathrm{t}-1$ et indicatrice temporelle de transition).

Champ : modèle multinomial : femmes âgées de 25 à 55 ans n'ayant pas été en congés maternité entre 2005 et 2008 ( $n=357759$, données empilées) ; modèle à effets fixes : femmes âgées de 25 à 55 ans entre 2005 et 2008 n'ayant pas été en congé maternité " movers " entre 2006 et $2007(n=38$ 152).

Source : Hygie (2005-2008). 